\title{
Radiative Hydromagnetic Shocks in Relativistic Outflow Sources
}

\author{
Jonathan Granot ${ }^{1}$ and Arieh Königl ${ }^{2}$ \\ ${ }^{1}$ Racah Institute, Hebrew University, Jerusalem 91904, Israel; jgranot@nikki.fiz.huji.ac.il \\ ${ }^{2}$ Department of Astronomy \& Astrophysics and Enrico Fermi Institute, University of \\ Chicago, 5640 S. Ellis Ave., Chicago, IL 60637; arieh@jets.uchicago.edu
}

\begin{abstract}
We calculate the structure of a relativistic shock wave in which the internal energy of the shocked fluid is radiated away on a time scale much shorter than the characteristic shock propagation time. The shock is assumed to move through a uniformly magnetized, neutral plasma consisting of protons, electrons, and positrons, and allowance is made for the possible production of electron/positron pairs in the shock itself. The radiation mechanism is taken to be synchrotron and inverse-Compton emission (the latter involving both synchrotron-produced and externally supplied seed photons) by the electrons and positrons. We simplify the discussion by considering a shock in which the magnetic field is transverse to the direction of propagation and focus attention on the properties of the radiative zone that forms behind the shock transition. In particular, we investigate the possibility that the compression induced by the cooling of the gas amplifies the magnetic pressure until it reaches (and ultimately exceeds) equipartition with the thermal pressure (which, in turn, limits the overall compression). We show that, if a significant fraction of the postshock thermal energy is deposited in the electron/positron component, then a considerable portion of the emitted radiation will come from regions of strong magnetic field even if the field immediately behind the shock transition is well below equipartition. This field amplification mechanism may be relevant to the production of synchrotron flares in blazars, miniquasars, and gamma-ray burst sources. We consider the latter application in some detail and show that this process may play a role in the prompt $\gamma$-ray and possibly also the optical "flash" and radio "flare" emission, but probably not in the afterglow.
\end{abstract}

Subject headings: galaxies: active - gamma rays: bursts - radiation mechanisms: nonthermal - shock waves 


\section{Introduction}

Propagating relativistic shock waves are the leading candidates for the origin of the emission from gamma-ray bursts (GRBs) and their afterglows (e.g., Piran 1999, 2000; Mészáros 2000) as well as of the spectral flares and apparent superluminal motions exhibited by the blazar class of active galactic nuclei (AGNs; e.g., Ulrich, Maraschi, \& Urry 1997) and their "miniquasar" Galactic counterparts (e.g., Mirabel \& Rodríguez 1999; Kaiser, Sunyaev, \& Spruit 2000). The shocks are thought to be associated with relativistic, jet-like outflows that are driven from the vicinity of a compact object: a massive black hole in blazars, a stellar-mass black hole in miniquasars, and a stellar-mass black hole or a rapidly rotating neutron star in GRBs. [3 In all cases except GRB afterglows, the shocks are believed to be internal, possibly resulting from the collision of "shells" (the products of nonsteady ejection episodes) inside the jet (e.g., Rees 1978; Fenimore et al. 1996; Sari \& Piran 1997b). In the case of GRB afterglows, the most common interpretation is in terms of shocks that form at the interface between the relativistically outflowing material and the surrounding medium (with the bulk of the observed emission arising in the "forward" shock that propagates into the ambient medium, whereas the "reverse" shock that is driven into the ejecta gives rise to an early "optical flash" and associated "radio flare"). The radiation in all three types of sources is inferred to be nonthermal, with the dominant emission mechanisms most commonly invoked being synchrotron and inverse Compton. In particular, both the $\gamma$-ray burst emission and the radio-through-X-ray afterglow radiation in GRBs, as well as the radio-through-X-ray emission in blazars and the radio-through-optical radiation in microquasars, has been attributed to synchrotron radiation (or, in certain models of GRBs, a closely related mechanism; e.g., Smolsky \& Usov 2000, Medvedev 2000, Lyutikov $\&$ Blackman 2001). In the case of blazars, the measurement of high ( $z 10 \%$ ) optical linear polarization in some objects directly supports the synchrotron interpretation (e.g., Angel \& Stockman 1980). In the case of GRBs there are already two confirmed detections of optical polarization (Covino et al. 1999; Wijers et al. 1999; Rol et al. 2000), which, despite the relatively low measured values $(\sim 1-3 \%)$, appear to be consistent with a synchrotron origin. In both blazars and microquasars the case for synchrotron radiation is supported also by measurements of radio polarization.

Synchrotron radiation requires the presence of charged particles (typically electrons or electron-positron pairs) with relativistic random velocities that move in a magnetic field. The magnitude of the energy densities of the random relativistic electron motions and of the

\footnotetext{
${ }^{3}$ In at least some GRBs there are, however, indications that the outflow may have a fairly large opening angle or even be spherical.
} 
magnetic field are parametrized in terms of their ratios to the total thermal energy density in the emission region by $\epsilon_{e}$ and $\epsilon_{B}$, respectively. In most cases, the values of these two parameters cannot be accurately determined from the observational data. However, in the case of blazars there are indications that the emitting gas is in approximate equipartition between the particle and magnetic energy densities (i.e., $\epsilon_{e} \approx \epsilon_{B}$ - e.g., Readhead 1994; Bower \& Backer 1998). This conclusion is also consistent with models of the nonthermal flares in microquasars (e.g., Kaiser et al. 2000). In the case of GRBs, if the observed $\gamma$-ray emission indeed represents synchrotron radiation, then considerations of radiative efficiency (based on estimates of source energetics) imply that $\epsilon_{e}$, and likely also $\epsilon_{B}$, are not much smaller than 1 (e.g., Sari \& Piran 1997a; Piran 1999). In the case of GRB afterglows, there have been a few instances in which data were available for deriving more direct estimates of these parameters. In particular, theoretical fitting of the broadband spectrum of the afterglow of GRB 970508 has yielded $\epsilon_{e} \sim 0.1-0.6$ and $\epsilon_{B} \sim 0.01-0.1$ (Wijers \& Galama 1999; Granot, Piran, \& Sari 1999; Chevalier \& Li 2000).

The inference that $\epsilon_{e}$ and $\epsilon_{B}$ are not much smaller than 1 in GRBs and at least some afterglows has important implications to the nature of the emitting regions in these sources. A comparatively large value of $\epsilon_{e}$ implies a high efficiency of shock acceleration of relativistic electrons in an electron-proton plasma or else a high electron-positron $\left(e^{ \pm}\right)$pair content in the postshock region (reflecting the composition of the preshock gas or arising from copious pair production in the shock). A comparatively large value of $\epsilon_{B}$ implies either a highly magnetized preshock medium or else an efficient mechanism of field amplification in (or behind) the shock. If $\epsilon_{e} \sim 1$ and the radiative cooling time is much shorter than the dynamical timef (which, for synchrotron radiation, would require also $\epsilon_{B}$ not to be too small), then the shock will be radiative: it will contain a radiative cooling layer, where most of the internal energy is radiated away, that will directly influence its dynamics. Nonrelativistic, thermally emitting shocks of this type are prevalent in the interstellar medium (e.g., Draine \& McKee 1993), but their properties in relativistic, nonthermal sources have not yet received much attention in the literature.

A radiative shock wave generally consists of a "shock transition," where the bulk of the kinetic energy of the preshock gas is dissipated, and a "radiative zone," where most of the dissipated energy is radiated away. The energy dissipation scale is typically much smaller than all other relevant length scales, so the shock transition can be treated as an infinitesimal front at the upstream end of the shock wave. The radiative zone, on the other

\footnotetext{
${ }^{4}$ The dynamical time is the time for a significant expansion of the emitting region, and is thus also the characteristic time for adiabatic energy losses. It is typically of the order of the shock distance from the origin divided by the instantaneous shock speed.
} 
hand, has a finite width, corresponding to the cooling length over which the postshock gas radiates away a significant fraction of the dissipated energy. After passing through the shock transition, the gas is usually compressed by a factor of a few (for Newtonian or mildly relativistic shocks), and in the absence of significant radiation this is the density enhancement that also characterizes the far-downstream (or postshock) region. However, it is a well-established result in the theory of interstellar shocks that the asymptotic compression can be far larger if the shock is radiative. This is a consequence of the fact that the total (thermal plus magnetic) pressure behind the shock transition remains roughly constant, so that, as long as magnetic effects are not important, the particle density $n$ increases inversely with decreasing temperature $T$.

A related important result for radiative interstellar shocks is that, if the gas has a "frozen in" magnetic field $B$ with a significant component $\left(B_{\perp}\right)$ perpendicular to the shock normal, then magnetic stresses become progressively more important as $T$ decreases behind the shock, a reflection of the fact that the magnetic pressure, associated with $B_{\perp}$, scales as $n^{2}$. If the preshock magnetic field is sufficiently strong, then the magnetic pressure in the cooling layer will reach equipartition with the thermal pressure at some point within the radiative zone. Beyond this point the gas will undergo little additional compression, and the cooling will proceed at nearly constant density rather than at constant thermal pressure (e.g., Hollenbach \& McKee 1979). Since the cooling time is proportional to $1 / n$ (a consequence of the fact that the cooling function $\Lambda$, the energy radiated per unit volume and per unit time, scales as $n^{2}$ for a thermally emitting gas), the inhibition of further compression by the magnetic stress results in a lower cooling efficiency in this part of the radiative zone.

One can anticipate that the basic properties of radiative hydromagnetic shocks in the interstellar medium (ISM) would carry over to the relativistic, nonthermal regime. In particular, one can expect a significant enhancement of the compression as well as of the downstream magnetic field in a radiative shock. The two cases are, however, expected to differ in detail. In particular, the cooling function of a synchrotron-emitting shocked gas satisfies $\Lambda \propto B^{2} n_{e} \propto n_{e}^{3}$ (assuming a predominantly transverse magnetic field behind the shock), where $n_{e}$ is the number density of electrons (and positrons). The explicit density dependence of $\Lambda$ is thus stronger than in a thermally emitting gas, which suggests that the dynamical influence of the magnetic field on the structure of the shock would be even more pronounced in the nonthermal case. This could have important implications to astrophysical sources in which such shocks occur. For one thing, it may provide a mechanism for amplifying the magnetic field up to equipartition strengths in cases where it is initially comparatively weak. Furthermore, as the processes of field amplification and synchrotron emission are mutually reinforcing in the radiative zone, this mechanism may 
naturally lead to synchrotron emission regions that are characterized by a high value of $\epsilon_{B}$. There is even the possibility, if inverse-Compton cooling also plays a role, that the bulk of the emitted synchrotron radiation would come from regions where the magnetic field is already at (or near) equipartition.?

In this paper we examine the aforementioned effects in a quantitative manner by studying the properties of relativistic, hydromagnetic, nonthermally emitting shocks. In 92 we describe the framework of our analysis and calculate the shock jump conditions. The structure of the radiative zone is considered in $\delta 3$. In $\$$ 国 we discuss the circumstances under which radiative shocks of this type are likely to occur in astrophysical sources, focusing on the case of GRBs and their afterglows. Our conclusions are presented in $\oint 5$.

\section{The Shock Transition}

\subsection{Formulation of the Problem}

We analyze the steady-state propagation of relativistic, planar, radiative shocks in a medium that contains a large-scale, ordered magnetic field. As we noted in $\S 1$, radiative shocks can be divided into a very thin "shock transition" and a "radiative zone" of finite thickness. We assume, for simplicity, that all the energy dissipation occurs in the shock transition and all the cooling in the radiative zone. The assumption of a stationary flow throughout the shock is justified by the fact that, by the definition of radiative shocks, the radiative cooling time $t_{\text {rad }}$ of the gas that passes the shock transition is much shorter than the shock dynamical time $t_{\text {dyn. }}$. The assumption of a plane-parallel flow is easily justified for the shock transition, and we now argue that it is also appropriate for the radiative zone.

The global shape of the shock is generally not planar. For example, if the shock is triggered by a nearly isotropic energy release at the source, as in supernova remnants and certain GRB scenarios, then it may assume an approximately spherical shape. The instantaneous distance of the shock front from the central source, $R$, is then given by the radius. If the geometry is not exactly spherical, then one needs to distinguish between the

\footnotetext{
${ }^{5}$ Relativistic radiative shocks have previously been considered in the context of blast-wave models (Blandford \& McKee 1976; Cohen, Piran, \& Sari 1998; Cohen \& Piran 1999). In particular, Cohen et al. (1998) and Cohen \& Piran (1999) obtained self-similar solutions for spherical blast waves that have adiabatic interiors and that are bounded by fully (or partially) radiative ultrarelativistic (or Newtonian) shocks. These studies incorporated the efect of radiative losses in a parametric way into the overall shock jump conditions. They did not, however, include specific cooling mechanisms and did not consider the detailed structure of the shock wave.
} 
distance $R$ and the local radius of curvature $R_{c}$. Typically, $R_{c} \sim R$ and both are much larger than the width of the radiative zone. For example, in a relativistic blast wave of Lorentz factor $\gamma \gg 1$, most of the energy and swept-up matter are concentrated within a thin shell of width $\Delta \sim R / \gamma^{2}$ in the observer frame, or $\Delta^{\prime}=\gamma \Delta \sim R / \gamma$ in the shock frame (e.g., Blandford \& McKee 1976). The width of the radiative zone is $\sim \Delta\left(t_{\text {rad }} / t_{\text {dyn }}\right) \ll \Delta \ll R$ in the observer frame, or $\sim \Delta^{\prime}\left(t_{\mathrm{rad}}^{\prime} / t_{\mathrm{dyn}}^{\prime}\right)=R_{c}^{\prime}\left(t_{\mathrm{rad}} / t_{\mathrm{dyn}}\right) \ll R_{c}^{\prime}$ in the shock frame (where $R_{c}^{\prime}=R / \gamma$ is the local radius of curvature in the shock frame). In any reasonable scenario,

$R_{c}^{\prime} \gtrsim \Delta^{\prime}$, and even for $R_{c}^{\prime} \sim \Delta^{\prime}$, the width of the radiative zone would still be smaller than $R_{c}^{\prime}$ by a factor $\sim t_{\text {rad }} / t_{\text {dyn }} \ll 1$. Given also that $R_{c}$ is not expected to change considerably on time scales shorter than the dynamical time, it is thus well justified to assume that the shock is planar when calculating the properties of the radiative zone.

We distinguish between the shock rest frame, in which we measure the time $t$, the distance behind the shock front $x$, and the fluid speed $v=\beta c$, and the fluid rest frame (the frame comoving with the local mean speed of the flow), in which we measure the thermodynamic quantities (the particle number density $n$, the rest-mass density $\rho$, the internal energy density $e$, the thermal pressure $p$, and the enthalpy density $w=\rho c^{2}+e+p$ ) as well as the magnetic field amplitude $B$. We simplify the treatment by assuming that the field is purely transverse, $B=B_{\perp}$, i.e., it only has a component that is perpendicular to the shock velocity and parallel to the shock front. This is justified by the fact that, upon traversing the shock, $B_{\perp}$ is amplified by a factor equal to the downstream-to-upstream density ratio (which can be quite large in a relativistic shock) whereas the parallel component remains unchanged, so that the postshock magnetic field is typically almost completely transverse.

\subsection{Shock Jump Conditions}

We denote quantities in the unshocked upstream region $(x<0)$ by the subscript 1 , whereas quantities immediately behind the shock transition are denoted by the subscript 2 . Quantities without numerical subscripts refer to the radiative zone. The shock is assumed to propagate in an electrically neutral plasma consisting of protons, electrons, and possibly also positrons. We define the parameter

$$
\chi \equiv \frac{n_{e^{+}}}{n_{e^{-}}}=\frac{n_{e^{+}}}{n_{p}+n_{e^{+}}},
$$

where $n_{p}, n_{e^{-}}$and $n_{e^{+}}$are the number densities of protons, electrons, and positrons, respectively. We assume that the number of protons is unchanged by the passage through the shock transition, but we allow the number of $e^{ \pm}$pairs to possibly increase in the shock 
transition (although not during the subsequent transit through the radiative zone). Thus we have

$$
u_{1} n_{p 1}=u_{2} n_{p 2}=u n_{p}, \quad \phi u_{1} n_{e 1}=u_{2} n_{e 2}=u n_{e}
$$

where $n_{e}=n_{e^{-}}+n_{e^{+}}$is the combined number density of electrons and positrons, $u=\gamma \beta$ is the proper speed (in units of the speed of light $c$ ), and the parameter $\phi$ accounts for the possible production of pairs in the shock. The constraint $\phi \geq 1$ implies that

$$
\chi_{2}=\frac{\phi\left(1+\chi_{1}\right)-\left(1-\chi_{1}\right)}{\phi\left(1+\chi_{1}\right)+\left(1-\chi_{1}\right)} \geq \chi_{1} .
$$

From equations (11) and (2) we infer

$$
n_{p 2}=\left(\frac{1-\chi_{1}}{1+\chi_{1}}\right) \frac{u_{1}}{u_{2}} n_{e 1} .
$$

With this parameterization it is straightforward to take the following limits: (i) no pair production at the shock corresponds to $\phi=1$; (ii) no protons corresponds to $\chi_{1}=\chi_{2}=1$; (iii) no pairs upstream corresponds to $\chi_{1}=0$.

The pressure and energy density of the magnetic field are given by $p_{B}=e_{B}=B^{2} / 8 \pi$, and hence the total pressure and enthalpy density are $p_{\text {tot }}=p+B^{2} / 8 \pi$ and $w_{\text {tot }}=w+B^{2} / 4 \pi$, respectively. In this paper we focus on the case in which the fluid is "cold" upstream and "hot" downstream. The former assumption means that the thermal pressure and internal energy density of the upstream fluid can be neglected $\left(e_{1}, p_{1} \ll \rho_{1} c^{2}\right)$, so that

$$
w_{1} \approx \rho_{1} c^{2}=n_{e 1} m_{e} c^{2}\left(\frac{m_{p}}{m_{e}} \frac{1-\chi_{1}}{1+\chi_{1}}+1\right),
$$

where $m_{p}$ and $m_{e}$ are the proton and electron masses, respectively. The latter assumption means that the shock Lorentz factor is sufficiently large for the random motions of the particles of the shocked fluid to be highly relativistic, so that, everywhere behind the shock transition,

$$
p_{p}=e_{p} / 3, \quad p_{e}=e_{e} / 3, \quad w=4 p=4 e / 3,
$$

where $p_{e}=p_{e^{+}}+p_{e^{-}}, p=p_{p}+p_{e}, e_{e}=e_{e^{+}}+e_{e^{-}}$, and $e=e_{p}+e_{e}$.

In the absence of a detailed shock model, it is necessary to specify how the postshock internal energy is divided between the proton and electron/positron components. We do this by parameterizing the ratio of the pressure of the electrons and positrons to that of the total thermal pressure just behind the shock transition,

$$
\eta \equiv \frac{p_{e 2}}{p_{2}} .
$$


In view of the assumed postshock equation of state (eq. [6]), $\eta$ is just the value of the parameter $\epsilon_{e}$ (see $\left.\S 1\right)$ immediately behind the shock transition $\left(\eta=\epsilon_{e 2}\right)$.

Under the assumption of ideal MHD, the magnetic flux is frozen into the fluid and remains constant in the shock frame. For a purely transverse field, this implies

$$
u_{1} B_{1}=u_{2} B_{2}=u B
$$

The momentum and energy conservation relations across the shock transition take the form

$$
\begin{aligned}
u_{1}^{2} w_{\mathrm{tot} 1}+p_{\mathrm{tot} 1} & =u_{2}^{2} w_{\mathrm{tot} 2}+p_{\mathrm{tot} 2} \\
\gamma_{1} u_{1} w_{\mathrm{tot} 1} & =\gamma_{2} u_{2} w_{\mathrm{tot} 2} .
\end{aligned}
$$

Since our main interest is in relativistic shock waves, we henceforth set $\beta_{1} \approx 1$ and $u_{1} \approx \gamma_{1} \gg 1$ and neglect the second term on the left-hand side of equation (9). Substitution of equations (5) and (6) into equations (9) and (10) then yields, after some algebra, an equation for $\beta_{2}$,

$$
\beta_{2}^{3}-\frac{5 u_{A 1}^{2}+4}{3\left(u_{A 1}^{2}+1\right)} \beta_{2}^{2}+\frac{1}{3} \beta_{2}+\frac{u_{A 1}^{2}}{3\left(u_{A 1}^{2}+1\right)}=0,
$$

and an equation for $p_{2}$,

$$
p_{2}=\frac{u_{1}^{2} u_{A 1}^{2} \rho_{1} c^{2}}{2 \delta_{2} u_{2}^{2}}
$$

where

$$
\delta_{2} \equiv \frac{B_{2}^{2}}{8 \pi p_{2}}=\frac{4 u_{2}^{2}+1}{2 u_{2}^{2} u_{A 1}^{-2}-1}
$$

is the ratio of the magnetic pressure to the thermal pressure just behind the shock transition, and $u_{A}^{2}=B^{2} / 4 \pi w$ is the square of the Alfvén proper speed (so $u_{A 1}^{2}=B_{1}^{2} / 4 \pi \rho_{1} c^{2}$ ). After the gas enters the radiative zone it cools and gets compressed, resulting in an increase in the magnetic-to-thermal pressure ratio. In fact, as we show in $\S 3.3, \delta(x)$ is a monotonically increasing function of $x$.

The solution of equation (11) is

$$
\beta_{2}=\frac{1+2 u_{A 1}^{2}+\sqrt{16 u_{A 1}^{2}\left(u_{A 1}^{2}+1\right)+1}}{6\left(u_{A 1}^{2}+1\right)} .
$$

In the limit $u_{A 1} \ll 1$ the magnetic effects near the shock transition are weak, and one recovers from equations (14) and (12) the expressions for a nonmagnetic, ultrarelativistic

\footnotetext{
${ }^{6}$ We check on the validity of this assumption at the end of $\S 3.2$.
} 
$\left(\beta_{1} \rightarrow 1\right)$ shock, $\beta_{2} \approx 1 / 3$ and $p_{2} \approx(2 / 3) u_{1}^{2} \rho_{1} c^{2}$. As $u_{A 1}$ is increased, $\beta_{2}$ becomes larger while $p_{2}$ becomes smaller. The dependence of $\beta_{2}, u_{2}$, and $\delta_{2}$ on $u_{A 1}$ is shown in Figure 1 . Note that, for a given shock speed, $u_{A 1}$ has an upper bound, namely $u_{1}$. This follows from the fact that, in order for a shock wave to exist, the relativistic Alfvén Mach number, given by $M_{A 1} \equiv u_{1} / u_{A 1}$ (e.g., Königl 1980), must exceed 1 .

\section{The Radiative Zone}

\subsection{Radiation}

We take the radiation mechanism behind the shock to be synchrotron emission and inverse-Compton scattering (IC hereafter) of seed photons by the relativistic electronpositron component. The seed photons may consist of internally emitted synchrotron photons, giving rise to synchrotron self-Compton radiation (SSC), or of an externally produced population of soft photons, in which case the IC emission is referred to as externalradiation Compton (ERC). Although we treat the postshock protons as a relativistic fluid, we neglect their (much weaker) nonthermal emission. ${ }^{7}$ Furthermore, we neglect any possible transfer of energy between the proton and electron/positron components in the radiative zone. Under these assumptions, the protons evolve adiabatically, and the fraction of the energy dissipated in the shock transition that can be radiated away is determined uniquely by the parameter $\eta$ defined in equation (7).

The radiating particles are assumed to have a monoenergetic energy distribution that can be characterized by a random (or "thermal") Lorentz factor $\gamma_{e}$ (the same for electrons and positrons) that is a function of the distance $x$ behind the shock. This approximation to the local energy distribution is appropriate for electrons and positrons that have undergone significant cooling. We note in this connection that observations and particle acceleration models of relativistic shock sources suggest that the energy distribution immediately behind the shock transition has the form of a power law. In this case the radiative zone is expected to consist of a thin layer just behind the shock transition where most of the electrons and positrons have not yet cooled significantly (and are thus not well approximated by a monoenergetic distribution), followed by a much wider layer where substantial cooling has already occurred and where the monoenergetic representation is adequate (see Granot,

7 If both the proton and electron components are effectively monoenergetic, then the ratio of their nonthermal (synchrotron or Thomson-scattering) cooling times behind the shock is $t_{\mathrm{rad}, p} / t_{\mathrm{rad}, e}=[\eta /(1-$ $\eta)]\left[\left(1-\chi_{2}\right) /\left(1+\chi_{2}\right)\right]\left(m_{p} / m_{e}\right)^{4}$, which is typically $\gg 1$. For a discussion of possible observable consequences in GRB sources of nonthermal emission by protons see, e.g., Zhang \& Mészáros (2001) and references therein. 
Piran, \& Sari 2000). Although the region where the monoenergetic approximation does not apply is relevant to the spectral modeling of the source, it can be safely neglected in a calculation of the cooling-zone dynamics.

The synchrotron emissivity (energy per unit volume per unit time measured in the rest frame of the fluid) from a population of relativistic electrons and positrons of total number density $n_{e}$ that have an isotropic velocity distribution is given by $\Lambda_{\text {syn }}=\sigma_{T} c n_{e} \gamma_{e}^{2} B^{2} / 6 \pi$ (e.g., Rybicki \& Lightman 1979), where $\sigma_{T}$ is the Thomson cross section. To obtain the total emissivity, one must add the IC component. The IC power of a single electron is $(4 / 3) \sigma_{T} c n_{e} \gamma_{e}^{2} e_{\mathrm{ph}}$, where $e_{\mathrm{ph}}$ is the energy density of photons, assuming an isotropic photon distribution. This is also the average IC power of an electron for an isotropic distribution of electron velocities, even when the photon distribution is not isotropic. Therefore, the total emissivity is

$$
\Lambda=\frac{4}{3} \sigma_{T} c n_{e} \gamma_{e}^{2}\left(e_{B}+e_{\mathrm{ph}}^{\mathrm{syn}}+e_{\mathrm{ph}}^{\mathrm{ext}}\right)
$$

where $e_{\mathrm{ph}}^{\mathrm{syn}}$ and $e_{\mathrm{ph}}^{\mathrm{ext}}$ are, respectively, the energy densities of the synchrotron and the external photons in the local frame.

We assume that the Thomson optical depth is much smaller than 1, a situation that often applies in astrophysical relativistic-jet sources. We further assume that the emitting region is optically thin to synchrotron self-absorption near the characteristic synchrotron frequency, which is typical in GRBs and their afterglows. Under these conditions, most of the radiated energy can freely escape the system. The average fractional energy gain, in a single electron scattering, of seed photons that propagate through the radiative zone is measured by the Compton $y$ parameter. In the Thomson limit $\left(\gamma_{e} h \nu \ll m_{e} c^{2}\right.$, where $\nu$ is the incident photon frequency in the local frame, i.e. the fluid rest frame), the average fractional energy gain of a seed photon moving at an angle $\theta=\cos ^{-1} \mu$ (measured in the shock frame) with respect to the $x$ direction is give by

$$
y(\mu)=\frac{4}{3} \int d \tau_{T} \gamma_{e}^{2}=\frac{4}{3} \frac{\sigma_{T}}{\mu} \int d x \gamma_{e}^{2} n_{e} \gamma(1-\beta \mu),
$$

where $d \tau_{T}=n_{e} \sigma_{T} d s$ is the differential optical depth for Compton scattering and $d s=\gamma(1-\beta \mu) d x / \mu$ is the differential of the distance traveled by the photon in the local frame. One can obtain $y$ by averaging the expression (16) over the angular distribution of the seed photons in the shock frame. In the case of an infinite planar shock, the term

\footnotetext{
${ }^{8}$ If these conditions are not satisfied, then the effective value of $t_{\text {rad }}$ is increased by opacity effects and the shock may not even be radiative. In any case, if the self-absorption frequency exceeds the characteristic frequency, the shock will become spectrally indistinguishable from a nonradiative shock (e.g., Granot et al. 2000).
} 
$1 / \mu$ that appears in equation (16) will cause the integral to diverge logarithmically for any angular distribution that does not vanish at $\mu=0$. However, under more realistic circumstances, one may expect the derived value of $y$ to remain finite. We approximate the integral in equation (16) by setting $\mu=1$ and then multiplying the value of the integrand just behind the shock transition by the characteristic size $x_{c}$ of the radiative zone (see eq. [20 below). This leads to

$$
y=\frac{4}{3} \sigma_{T} \gamma_{e 2}^{2} n_{e 2} \gamma_{2}\left(1-\beta_{2}\right) k_{1} x_{c}
$$

where we have introduced a numerical factor $k_{1}$ (expected to be of order unity) that incorporates the various uncertainties involved in our approximation. For $y \ll 1$ only single scatterings are important, whereas for $y \gtrsim 1$ multiple scatterings can significantly affect the energy and spectrum of the scattered photons. However, a typical seed photon with initial energy $h \nu$ is upscattered on the average to an energy $\sim \gamma_{e}^{2} h \nu$ in a single scattering. Usually, the typical electron Lorentz factor $\gamma_{e}$ is sufficiently high for the Thomson limit to no longer apply even in the second scattering $\left(\gamma_{e}^{3} h \nu>m_{e} c^{2}\right)$. This causes the energy gain in each successive scattering to be significantly reduced on account of the Klein-Nishina decrease in the cross section and of electron recoil. We therefore neglect multiple scatterings in our analysis.

The energy density $e_{\mathrm{ph}}^{\mathrm{syn}}$ of the synchrotron photons may be calculated in a manner analogous to that of the Compton $y$ parameter and is subject to similar uncertainties. We treat it in the same manner as above by introducing a new parameter of order unity, $k_{2}$ :

$$
e_{\mathrm{ph}}^{\mathrm{syn}}=\Lambda_{\mathrm{syn}} \frac{x_{c}^{\prime}}{c}=\frac{4}{3} \sigma_{T} n_{e 2} \gamma_{e 2}^{2} \gamma_{2}\left(1-\beta_{2}\right) k_{2} x_{c}=\frac{k_{2}}{k_{1}} y\left(\frac{B_{2}^{2}}{8 \pi}\right),
$$

where $x_{c}^{\prime}=x_{c} \gamma(1-\beta)$ is the length of the trajectory of a photon in the $x$ direction $(\theta=0)$ within the radiative zone, as measured in the comoving frame. We also consider an external photon distribution that is isotropic and has an energy density $e_{\mathrm{ph} 1}^{\mathrm{ext}}$ in the frame of the preshock fluid. In the local rest frame of the shocked fluid, the energy density of the external photons is given by

$$
\frac{e_{\mathrm{ph}}^{\mathrm{ext}}}{e_{\mathrm{ph} 1}^{\mathrm{ext}}}=\gamma_{r}^{2}\left(1+\frac{\beta_{r}^{2}}{3}\right) \approx \frac{4}{3} \gamma_{1}^{2} \gamma^{2}(1-\beta)^{2} \approx \frac{4}{3} \gamma_{1}^{2} \gamma_{2}^{2}\left(1-\beta_{2}\right)^{2}
$$

(e.g., Rybicki \& Lightman 1979), where $\gamma_{r}=\gamma_{1} \gamma\left(1-\beta_{1} \beta\right) \approx \gamma_{1} \gamma(1-\beta)$ is the relative Lorentz factor between the shocked and unshocked fluids.

The cooling length $x_{c}$ is the distance behind the shock transition over which the electrons/positrons lose the bulk of their internal energy; it is thus the characteristic size of the radiative zone. Since a significant fraction of the internal energy must be radiated 
away before the hydrodynamic quantities start to deviate significantly from their values just behind the shock transition, a substantial portion of the radiation would be emitted under conditions that prevail at the upstream end of the radiative zone. One can therefore evaluate $x_{c}$ by using the parameter values at $x_{2}$,

$$
x_{c} \equiv \frac{u_{2} c e_{e 2}}{\Lambda_{2}}=\frac{2 \pi m_{e}^{2} c^{4} u_{2} n_{e 2}}{(1+b+k y) \sigma_{T} B_{2}^{2} p_{e 2}}=\frac{16 \pi^{2} m_{e}^{2} c^{4} n_{e 1}}{(1+b+k y) \sigma_{T} B_{1}^{4}} \frac{u_{2}^{4}}{u_{1}^{3}} \frac{\phi \delta_{2}}{\eta},
$$

where $k \equiv k_{2} / k_{1}$ and $b \equiv e_{\mathrm{ph} 2}^{\mathrm{ext}} / e_{B 2}$, , ] and where we have used equations (15) and (18). From equations (20) and (17) we obtain an equation for $y$ :

$$
(k y)^{2}+(1+b) k y=k_{2} u_{2} \gamma_{2}\left(1-\beta_{2}\right) \frac{e_{e 2}}{e_{B 2}}=k_{2} u_{2} \gamma_{2}\left(1-\beta_{2}\right) \frac{3 \eta}{\delta_{2}} \equiv a,
$$

which yields

$$
k y=\frac{(1+b)}{2}\left(\sqrt{1+\frac{4 a}{(1+b)^{2}}}-1\right) \approx\left\{\begin{array}{cc}
\sqrt{a} & 1, b^{2} \ll a \\
a & a, b \ll 1 \\
a / b & 1, a \ll b^{2}
\end{array}\right.
$$

In the first two limits the external photons are not important, and we obtain the familiar expressions for emission dominated by SSC and synchrotron, respectively (Sari, Narayan, \& Piran 1996). The physical meaning of each of these limits can be understood by comparing the relative magnitudes of the different components of the energy density, keeping in mind that $a \approx e_{e 2} / e_{B 2}, b=e_{\mathrm{ph} 2}^{\mathrm{ext}} / e_{B 2}$, and $e_{\mathrm{ph} 2}^{\mathrm{syn}} \approx y e_{B 2}$. In the first limit $y \approx a^{1 / 2} \gg 1$ and $b^{2} \ll a$, implying that $e_{B 2}, e_{\mathrm{ph} 2}^{\mathrm{ext}} \ll e_{\mathrm{ph} 2}^{\mathrm{syn}}$, which according to equation (15) means that most of the radiated power goes into $\mathrm{SSC}$. One also obtains $e_{e 2} \approx y e_{\mathrm{ph} 2}^{\mathrm{syn}}$, which is consistent with this interpretation, since, in a steady-state radiative shock, the incoming flux of internal energy $\left(\beta_{2} c e_{e 2} \approx c e_{e 2}\right)$ equals the outgoing radiation flux, which in this limit is dominated by $\mathrm{SSC}$ and is therefore approximately equal to $c y e_{\mathrm{ph} 2}^{\mathrm{syn}}$. In the second limit $y \approx a \ll 1$ and $b \ll 1$, implying that $e_{\mathrm{ph} 2}^{\mathrm{ext}}, e_{\mathrm{ph} 2}^{\mathrm{syn}} \ll e_{B 2}$, which means that synchrotron radiation is dominant over IC. In this case we also have $e_{e 2} \approx e_{\mathrm{ph} 2}^{\mathrm{syn}}$, which implies that all the internal energy goes into synchrotron radiation. In the third limit $y \approx a / b$ and $1, a \ll b^{2}$, implying $e_{\mathrm{ph} 2}^{\mathrm{syn}}, e_{B 2} \ll e_{\mathrm{ph} 2}^{\mathrm{ext}}$, which means that most of the emitted power goes into IC-scattered external photons (ERC). This conclusion is consistent with the fact that $e_{e 2} \approx y e_{\mathrm{ph} 2}^{\mathrm{ext}}$ in this case, which means that all the internal energy is going into ERC. Note that the value of $y$ ceases to be

\footnotetext{
${ }^{9}$ Note that the parameter $b$ is also approximately equal to $e_{\mathrm{ph} 1}^{\mathrm{ext}} / e_{B 1}$, as both energy densities increase by a factor $\sim \gamma_{1}^{2}$ across the shock transition (see eqs. [8] and [19]).
} 
pertinent in this limit as it no longer gives the ratio of the IC and synchrotron emissivities (in particular, $y$ could be $\ll 1$ even as IC remains dominant).

The photon energy densities $e_{\mathrm{ph}}^{\mathrm{ext}}$ and $e_{\mathrm{ph}}^{\mathrm{syn}}$ are approximately constant throughout the radiative zone, whereas, as we demonstrate in $\$ 3.3$, the magnetic energy density $e_{B} \propto B^{2}$ may be considerably amplified if $\eta \approx 1$. Thus, even if synchrotron emission is not dominant immediately behind the shock transition (as in limits 1 and 3 above), it may become so further downstream. However, by the time this happens, only a small fraction of the initial internal energy would typically be left in the electron/positron component. Therefore, the total emission would still be dominated by IC (either SSC, as in the first limit, or ERC, as in the third limit). Nevertheless, given that the characteristic IC frequency is usually much higher than that of the synchrotron emission (by a factor $\sim \gamma_{e}^{2}$ in the case of SSC), it is likely even in this case that only the synchrotron component is detected when the observation frequency is not too high.

\subsection{Hydrodynamics}

The steady-state momentum and energy conservation equations in the shock frame can be written as

$$
\begin{aligned}
\partial_{x}\left(u^{2} w_{\text {tot }}+p_{\text {tot }}\right) & =-u \Lambda / c, \\
\partial_{x}\left(\gamma u w_{\text {tot }}\right) & =-\gamma \Lambda / c .
\end{aligned}
$$

The terms on the right-hand sides of these equations represent the contribution of the radiation field to the momentum and energy fluxes, respectively. Their forms are appropriate when the emitted or scattered radiation exerts no net force on the fluid; in particular, they apply when the radiation field is isotropic in the fluid frame. If the magnetic field is ordered, as we have assumed, then the synchrotron emission of any given particle is not isotropic in the fluid rest frame; however, so long as the electron/positron rest-frame velocity distribution is isotropic, the total synchrotron radiation will carry no net momentum flux in this frame and will, therefore, exert no net force on the fluid. The resulting synchrotron photon energy density (eq. [18]), when scattered by the isotropic electron/positron distribution, gives rise to an SSC emissivity that is isotropic in the fluid frame. The same applies to an ERC component that arises from the scattering (by electrons/positrons that are isotropic in the local rest frame) of an isotropic (in the rest

\footnotetext{
${ }^{10}$ Note that the corresponding terms in equations (32) and (33) of Cohen et al. (1998) evidently need to be corrected.
} 
frame of the central source) external radiation field (where now the photon energy density that appears in the isotropic-emission expression, in the local rest frame, is given by eq. [19]; see Dermer \& Schlikeiser 1994).⿴囗十

From equations (23) and (24) one obtains an equation for the entropy:

$$
w_{\text {tot }} \partial_{x} u+u \partial_{x}\left(w_{\text {tot }}-p_{\text {tot }}\right)=-\Lambda / c
$$

The magnetic field evolves according to equation (8) and the protons evolve adiabatically,

$$
\frac{p_{p}}{p_{p 2}}=\left(\frac{n_{p}}{n_{p 2}}\right)^{4 / 3}=\left(\frac{u}{u_{2}}\right)^{-4 / 3}
$$

so the terms that involve them in equation (25) cancel out. One is left with

$$
4 p_{e} \partial_{x} u+3 u \partial_{x} p_{e}=-\Lambda / c
$$

Eliminating the radiative terms from equations (23) and (24) results in

$$
\frac{d p}{d u}=\frac{B_{2}^{2} u_{2}^{2}}{4 \pi u^{3}\left(1+u^{2}\right)}-\frac{4 u p}{\left(1+u^{2}\right)},
$$

or, in terms of the dimensionless pressure $\tilde{p} \equiv p / p_{2}$,

$$
\frac{d \tilde{p}}{d u}=\frac{2 \delta_{2} u_{2}^{2} u^{-3}-4 u \tilde{p}}{\left(1+u^{2}\right)}
$$

Equation (29) can be integrated to give

$$
\tilde{p}(u)=\frac{\left(1+u_{2}^{2}\right)^{2}+\delta_{2}\left[1-\left(u_{2} / u\right)^{2}+2 u_{2}^{2} \ln \left(u / u_{2}\right)\right]}{\left(1+u^{2}\right)^{2}} .
$$

Using equations (30), (26), and ([7), we deduce

$$
\begin{gathered}
\tilde{p}_{e}(u)=\frac{\tilde{p}-(1-\eta) \tilde{p}_{p}}{\eta}= \\
\frac{\left(1+u_{2}^{2}\right)^{2}+\delta_{2}\left[1-\left(u_{2} / u\right)^{2}+2 u_{2}^{2} \ln \left(u / u_{2}\right)\right]}{\eta\left(1+u^{2}\right)^{2}}-\frac{1-\eta}{\eta}\left(\frac{u_{2}}{u}\right)^{4 / 3}
\end{gathered}
$$

\footnotetext{
${ }^{11}$ We ignore in our discussion the effect of the radiation drag force (corresponding to a nonvanishing momentum flux of intercepted photons in the fluid rest frame) that is exerted by an external radiation field on the bulk motion of the fluid. This is consistent with our neglect of any variations in the gross properties of the shock that occur on time scales $z t_{\mathrm{dyn}}$.
} 
where $\tilde{p}_{p} \equiv p_{p} / p_{p 2}$ and $\tilde{p}_{e} \equiv p_{e} / p_{e 2}$. We already have explicit expressions for $p_{e}, p_{p}, B, n_{p}, n_{e}$, and $\gamma_{e}=3 p_{e} / n_{e} m_{e} c^{2}$ as functions of $u$. To obtain their values as a function of the distance behind the shock, we need to know $u(x)$. Introducing the dimensionless variable $\xi \equiv x / x_{c}$ and using equations (12), (15), (20), (27), and (28), we get

$$
\frac{d u}{d \xi}=-\frac{3 \eta u_{2}^{2} u\left(1+u^{2}\right) \tilde{p}(u)_{e}^{2}\left[(1+Y) /\left(1+Y_{2}\right)\right]}{4\left(1-2 u^{2}\right)\left[(1-\eta) u_{2}^{4 / 3} u^{2 / 3}+\eta u^{2} \tilde{p}(u)_{e}\right]+6 \delta_{2} u_{2}^{2}},
$$

where the function

$$
Y(u)=(b+k y)\left(\frac{u}{u_{2}}\right)^{2}
$$

represents the ratio of the local IC (including SSC and ERC) and synchrotron emissivities, and $Y_{2}=(b+k y)$ is the value of $Y$ just behind the shock transition. Note that the only dependence of the function $Y(u)$ on the Compton $y$ parameter (see eq. [17]) is through the combination $k y$, which, in turn, is given (in terms of the parameters $a$ and $b$ ) by equation (22). As both $u_{2}$ and $\delta_{2}$ are functions of $u_{A 1}$ (see eqs. [13 and 14]), $a$ is determined by the parameters $k_{2}, u_{A 1}$, and $\eta$. For simplicity, we set $k_{2}=1$ in the rest of this paper. Therefore, $Y(u)$ and the right-hand side of equation (32) are specified by the parameters $\eta, u_{A 1}$, and $b$, and $u(\xi)$ can be obtained by solving equation (32) numerically, using the boundary condition $u(\xi=0)=u_{2}$.

Before proceeding to present the solutions of the above set of equations, we digress briefly to check on the validity of the ideal-MHD approximation that underlies our analysis. Under the assumption of ideal MHD, the electric field vanishes in the rest frame of the fluid. The magnetic field in the shock frame is then given by $\gamma \vec{B}=u_{1} B_{1} \hat{e}_{y} / \beta(x)$, where we have chosen the $y$ axis to point along the direction of the magnetic field. This implies a current density

$$
\vec{j}=\frac{c}{4 \pi} \nabla \times(\gamma \vec{B})=-\frac{u_{1} B_{1} c}{4 \pi \beta^{2}} \frac{d \beta}{d x} \hat{e}_{z} .
$$

Since in our case $\vec{j}$ is perpendicular to the direction of the velocity, it has the same magnitude in the shock frame. In order for the ideal-MHD approximation to be valid, the current density in the fluid rest frame cannot exceed $j_{\max }=n e c=n_{2} u_{2} e c / u(x)$, where $n=n_{e}+n_{p}$ is the total number density of charged particles (e.g., Melatos \& Melrose 1996). Furthermore, so long as $j \ll j_{\max }$, the effect of anomalous resistivity associated with current-driven plasma instabilities is expected to be small (e.g., Spruit, Daigne, \& Drenkhahn 2001). The condition $j<j_{\max }$ translates into

$$
\frac{\gamma}{\beta}\left|\frac{d \beta}{d x}\right|<\frac{4 \pi e n_{2}}{B_{2}} .
$$


In the radiative zone $(\gamma / \beta)|d \beta / d x| \lesssim \gamma_{2} / x_{c} \approx 1 / x_{c}$, so equation (35) may be written as

$$
1<\frac{4 \pi e n_{2} x_{c}}{B_{2}}=\frac{64 \pi^{3} m_{e}^{2} c^{4} e}{\sigma_{T}} \frac{n_{1} n_{e 1} u_{2}^{4}}{B_{1}^{5} u_{1}^{3}}\left(\frac{n_{2} u_{2}}{n_{1} u_{1}}\right) \frac{\phi \delta_{2}}{\eta\left(1+Y_{2}\right)} .
$$

Note that this inequality can be rewritten in terms of the postshock electron Larmor radius $r_{\mathrm{L}, 2}$ as $r_{\mathrm{L}, 2}<3 x_{c} / 2 \delta_{2}$. This shows that, if ideal MHD holds in the radiative zone, then the condition for the fluid approximation to be valid there (essentially $r_{\mathrm{L}, 2} \ll x_{c}$ ) will also be satisfied. We now check whether the inequality (36) holds under the most unfavorable choice of parameters. The latter is obtained by taking $\eta=\phi=1$ and $\delta_{2} \ll 1$, which implies $n_{2} u_{2}=n_{1} u_{1}, u_{A 1} \ll 1, u_{2} \approx 1 / \sqrt{8}$, and $\delta_{2} \approx 3 B_{1}^{2} / 2 \pi \rho_{1} c^{2}$, resulting in

$$
1<\frac{3 \pi^{2} m_{e}^{2} c^{2} e}{2 \sigma_{T}\left(1+Y_{2}\right)} \frac{n_{e 1} n_{1}}{\rho_{1} B_{1}^{3} u_{1}^{3}}
$$

The minimal value of $n_{e 1} / \rho_{1}$ is $1 / m_{p}$, which is obtained for $\chi_{1}=0$. This leads to

$$
1<\frac{3 \pi^{2} m_{e}^{2} c^{2} e}{2 \sigma_{T} m_{p}\left(1+Y_{2}\right)} \frac{n_{1}}{B_{1}^{3} u_{1}^{3}}=\frac{9.5 \times 10^{6} n_{p 1,0}}{\left(1+Y_{2}\right) B_{1,0}^{3} u_{1}^{3}} \approx \frac{2.2 \times 10^{8} n_{p 1,0}}{\left(1+Y_{2}\right) B_{2,0}^{3}},
$$

where here and below numerical subscripts (such as the subscript 0 in $n_{p 1,0}$ ) refer to powers of 10 in c.g.s units.

As an illustration we apply this condition to GRBs, considering both the prompt high-energy emission (resulting from internal shocks within the flow) and the lower-energy afterglow (arising from the interaction of the flow with the surrounding medium). We begin with internal shocks. We use the conventional parameterization $B_{2}^{2}=8 \pi \epsilon_{B 2} e_{2}$ (where we identify $\epsilon_{B 2}=\delta_{2} / 3$ ), and write (e.g., Piran 1999) $e_{2}=\left(\gamma_{12}-1\right)\left(4 \gamma_{12}+3\right) n_{p 1} m_{p} c^{2}$, $n_{p 1}=E / 4 \pi m_{p} R^{2} \gamma^{2} c^{3} t_{\text {dur }}$, and $R=2 \gamma^{2} c t_{\text {dur }} \zeta$, where $E$ is the total energy deposited in the flow, $\gamma$ is the typical Lorentz factor of the flow with respect to the central object (and the observer), $\gamma_{12}$ is the relative Lorentz factor between the shocked and the unshocked fluids, $t_{\text {dur }}$ is the duration of the burst, and $\zeta=\delta t / t_{\text {dur }}$ is a measure of the variability of the burst, with $\delta t$ being the smallest time scale for significant variation during the burst. Thus we obtain

$$
1<\frac{1.3 \times 10^{5} t_{\mathrm{dur}, 1}^{3 / 2} \gamma_{2}^{3} \zeta_{-2}}{\left(1+Y_{2}\right) \epsilon_{B 2}^{3 / 2} E_{52}^{1 / 2}\left[\left(\gamma_{12}-1\right)\left(4 \gamma_{12}+3\right)\right]^{3 / 2}} \approx \frac{3.6 \times 10^{3} t_{\mathrm{dur}, 1}^{3 / 2} \gamma_{2}^{3} \zeta_{-2}}{\left(1+Y_{2}\right) \epsilon_{B 2}^{3 / 2} E_{52}^{1 / 2}}
$$

where we used $\gamma_{12}=2$ on the right-hand side (consistent with the expectation that $\gamma_{12}$ is of the order of 1). Turning now to the afterglow stage of GRBs, we evaluate the inequality (37) by using $e_{2}=4 \gamma^{2} n_{p 1} m_{p} c^{2}$ in the parametrized expression for $B_{2}$. This yields

$$
1<1.2 \times 10^{2}\left(1+Y_{2}\right)^{-1} \epsilon_{B 2}^{-3 / 2} n_{p 1,3}^{-1 / 2} \gamma_{2}^{-3}
$$


where we scaled $\gamma$ and $n_{p 1}$ by their rough upper limits for typical afterglows. (The Lorentz factor $\gamma$ decreases during the afterglow evolution, as does $n_{p 1}$ if the shock propagates into a stellar-wind environment.) Since the factor $\left(1+Y_{2}\right) \epsilon_{B 2}^{3 / 2}$ in equations (39) and (40) never exceeds 1 in the absence of a strong external radiation field (being approximately equal to $\max \left\{\epsilon_{B 2}^{3 / 2}, \epsilon_{B 2} \eta^{1 / 2}\right\}$; see eq. 22]), we conclude that the use of the MHD approximation for studying the cooling layers behind radiative shocks should be well justified throughout the evolution of typical GRBs and their afterglows.

\subsection{Results}

At large distances behind the shock $(\xi \gg 1)$ the electrons and positrons are left with only a small fraction of their internal energy, and all hydrodynamic quantities approach some constant asymptotic value: $u \approx u_{\min }, B \approx B_{\max }=B_{2} u_{2} / u_{\min }$. By equating the radiative cooling time with the flow time, one infers that $\gamma_{e} \approx \gamma_{e 2} / \xi$ in that region (e.g., Granot et al. 2000). Also, $n_{e} \approx n_{e 2} u_{2} / u_{\min }$, and hence $\tilde{p}_{e} \approx u_{2} / u_{\min } \xi$ for $\xi \gg 1$. The fact that $\tilde{p}_{e}(\xi)>0$ for any finite value of $\xi$ implies that $u(\xi)>u_{\min }$, so $u$ becomes equal to $u_{\min }$ only asymptotically. One can determine $u_{\text {min }}$ by setting equation (31) equal to zero and (numerically) solving for $u$ : this yields $u_{\min }$ as a function of $\eta$ and $u_{A 1}$. In Figures 2 and 3 we fix one of these variables and show $u_{\text {min }}$, normalized by $u_{2}$, as a function of the other variable. In the limit $\eta \rightarrow 0$ there are no radiation losses, so the speed of the shocked fluid does not change: $u_{\min }=u_{2}$. In the limit $\eta \rightarrow 1$ the electrons and positrons contain all the postshock internal energy and radiate it away, and the attendant compression eventually renders the magnetic pressure dominant. Since the magnetic field is amplified by a factor $\sim u_{2} / u_{\text {min }}$ in the radiative zone (see eq. [8]), a smaller initial magnetic field (smaller $u_{A 1}$ ) results in a lower value of $u_{\min } / u_{2}$. Furthermore, the total pressure, $p_{\text {tot }}$, is approximately constant throughout the radiative zone, and for $u_{A 1} \ll 1$ it becomes independent of $u_{A 1}$ $\left(p_{\text {tot }} \approx p_{2} \approx(2 / 3) u_{1}^{2} \rho_{1} c^{2}\right)$. Since in the limit $\eta \rightarrow 1$ the magnetic pressure eventually becomes dominant $\left(B_{\max }^{2} / 8 \pi \approx p_{\text {tot }}\right), B_{\max }$ also becomes independent of $u_{A 1}$ for $u_{A 1} \ll 1$, and we obtain $u_{\min } / u_{2}=B_{2} / B_{\max } \propto u_{A 1}$, as can be seen in Figure 3 .

Figure 4 displays $u(\xi)$, normalized by $u_{2}$, for $\eta=0.9, b=0$, and several values of $u_{A 1}$. For large values of $u_{A 1}(z 0.08)$ the asymptotic value $u=u_{\min }$ (shown by a dashed line) is approached at smaller values of $\xi$ (traced by the asterisks) as $u_{A 1}$ decreases, whereas the converse occurs for smaller values of $u_{A 1}$. This can be understood as follows. For large values of $u_{A 1}$ the initial magnetic field $B_{2}$ is strong and $Y_{2}$ is close to 1 , so synchrotron cooling is important early on. As the internal energy is radiated, the fluid becomes denser and the magnetic field grows stronger, which enhances the synchrotron emission. Since 
the cooling length is calculated by using the initial synchrotron emissivity, the value of $x_{c}$ is overestimated in this case and hence the asymptotic value of $u=u_{2}$ is approached at lower values of $\xi$. This effect is stronger the larger the value of $B_{\max }$, which for a fixed $\eta$ is obtained for lower values of $u_{A 1}$. However, for sufficiently small values of $u_{A 1}, Y_{2}$ comes to exceed 1 and IC becomes the dominant cooling mechanism. For $u_{A 1} \ll 1$ synchrotron radiation hardly affects the dynamics, and $x_{c}$ is determined by the IC emissivity just behind the shock transition. Since, unlike the magnetic energy density, the photon energy density that determines the IC cooling rate does not increase with $\xi$, the value of $x_{c}$ better approximates the actual cooling length, and consequently $u_{\min }$ is approached at larger values of $\xi$ for smaller $u_{A 1}$.

Figure 5 shows $u(\xi)$, normalized by $u_{2}$, for $u_{A 1}=0.05, b=0$, and several values of $\eta$. In this case the asymptotic value of $u$ is approached at smaller values of $\xi$ for larger values of $\eta$, which correspond to larger values of $B_{\max }$ and a stronger enhancement of the synchrotron cooling.

It is straightforward to verify from equations (31) and (32) that $p_{e}$ is a monotonically increasing function of $u$ and that $u$ is a monotonically decreasing function of $x$. Therefore, as expected, both $p_{e}$ and $u$ decrease the further one gets from the shock front.

To demonstrate the evolution of the various components of the pressure, we show in Figure 6 the dependence of $p, p_{e}, p_{p}, p_{B}$ and $p_{\text {tot }}$ (each normalized by $p_{2}$ ) on $u$ for $\eta=0.9$ and $u_{A 1}=0.2$. Equipartition occurs when the total thermal pressure equals the magnetic pressure, $p\left(u_{\mathrm{eq}}\right)=p_{B}\left(u_{\mathrm{eq}}\right)$, with $u_{\mathrm{eq}}$ denoting the value of $u$ at equipartition. However, this condition is not satisfied in all cases. There is a critical value of $u_{A 1}$, which we denote by $u_{\text {eq }}$, for which $\delta_{2}\left(u_{\text {eq }}\right)=1$. We find that $u_{\text {eq }}=0.447$. For $u_{A 1}>u_{\text {eq }}$ the magnetic field just behind the shock transition is already above equipartition, $B_{2}>B_{\text {eq2 }}$, where $B_{\text {eq }}$ is given by $B_{\text {eq }}=(8 \pi p)^{1 / 2}$. On the other hand, $B_{2}<B_{\text {eq2 }}$ for $u_{A 1}<u_{\text {eq }}$, and only if $\eta$ is above some critical value $\eta_{\min }\left(u_{A 1}\right)$ will the asymptotic magnetic field be above equipartition. Since $u_{2}$, and therefore also $\delta_{2}$, are functions of $u_{A 1}, \tilde{p}$ is a function only of $u_{A 1}$ and $u$ (see eq. [30]). Therefore, $\eta_{\min }\left(u_{A 1}\right)$ may be found by (numerically) solving the equation

$$
\tilde{p}\left[u_{A 1}, u_{\min }\left(u_{A 1}\right)\right]=\delta_{2}\left(u_{A 1}\right)\left[\frac{u_{2}\left(u_{A 1}\right)}{u_{\min }\left(u_{A 1}\right)}\right]^{2},
$$

where $u_{\min }\left(u_{A 1}\right)=u_{\min }\left[\eta_{\min }\left(u_{A 1}\right), u_{A 1}\right]$. Figures 7 and 8 show $\eta_{\min }$ as a function of $u_{A 1}$ and $\delta_{2}$, respectively. The critical value of $\eta$ is close to 1 for small values of $u_{A 1}$ (or $\left.\delta_{2}\right)$ since, in this case, the initial magnetic field is small, and almost all of the internal energy needs to be lost in order for $B_{\max } / B_{2}\left(=u_{2} / u_{\min }\right)$ to be large enough for $B_{\max }$ to reach equipartition.

As we noted in $\S 1$, there are direct observational indications that at least in some 
relativistic shock sources the magnetic field is not far below equipartition. Using the calculated radiative shock structure, we can address the question of how far from equipartition the magnetic field is at the location where the bulk of the observed radiation is emitted. A fluid element starts radiating just behind the shock transition, where $B=B_{2}$ and $\delta=\delta_{2}$, and as it flows downstream the magnetic field increases, and so does the magnetic-to-thermal pressure ratio $\delta$. Thus, even if initially the magnetic field were much below equipartition $\left(\delta_{2} \ll 1\right)$, it would be amplified by a large factor $\left(\delta \gg \delta_{2}\right)$ in the radiative zone and might eventually even exceed equipartition $(\delta>1)$. It is therefore conceivable that a considerable fraction of the radiation could be emitted from a region where the magnetic field is much larger than the immediate-postshock value. We now examine this possibility in a quantitative manner.

Since the internal energy of the electrons and positrons is characterized by their thermal Lorentz factor $\gamma_{e} \propto p_{e} / n_{e} \propto u p_{e}$, their fractional remaining energy is given by $u p_{e}(u) / u_{2} p_{e 2}=u \tilde{p}_{e}(u) / u_{2}$. Figure 9 shows this quantity as a function of $\delta_{2}$ at two different instants: (i) at equipartition, when $\delta=1$, and (ii) when $\delta=0.01$. For $\eta$ close to 1 a significant fraction of the internal energy of the electrons and positrons is still left when the field reaches equipartition even if $B_{2} \ll B_{\text {eq2 }}$. In this case a significant portion of the radiation originates in a region where the magnetic field is close to equipartition. We find that, so long as the energy dissipated in the shock transition is deposited mainly in the $e^{ \pm}$ component (i.e., $\eta \approx 1$ ), then, even if $\delta_{2}$ is as small as $\sim 10^{-4}-10^{-3}$, a considerable fraction of the radiation is still emitted at $\delta>10^{-2}$. Note that, for $\eta \approx 1$ and $\delta_{2} \ll 1, a \sim 1 / \delta_{2} \gg 1$ (see eq. [21]), so that, in the absence of a strong external photon field $\left(b \ll a^{1 / 2} \sim \delta_{2}\right)$, $y \approx a^{1 / 2} \sim \delta_{2}^{-1 / 2} \gg 1$ (see eq. [22]). This implies that SSC dominates the synchrotron emission, with the synchrotron component contributing only a fraction $1 /(1+y) \sim \delta_{2}^{1 / 2} \ll 1$ of the total radiation. However, as we noted at the end of $\S 3.1$ (see also $\S$ ), the synchrotron emission may dominate the SSC contribution in the observed frequency range, in which case a significant fraction of the observed radiation could, in fact, originate from a region where $\delta \gg \delta_{2}$.

In Figure 10 we show the fraction of the total synchrotron emission that is emitted above a given value of $B / B_{\text {eq }}$, as well as the fraction of the total emission contributed by the synchrotron process above that value of $B / B_{\text {eq }}$, as a function of the magnetic field strength behind the shock. Three sets of parameters are shown, labeled A through $\mathrm{C}$, which correspond to the three limits (from top to bottom, respectively) of equation (22). In case A, SSC is dominant near the shock front, whereas further downstream the magnetic field is strongly amplified by compression, and synchrotron emission becomes dominant. Altogether, synchrotron emission constitutes $45 \%$ of the total emission, whereas near the maximal field amplitude $\left(B \approx B_{\max }\right)$ the synchrotron process contributes $94 \%$ of the total 
emission. A large fraction of the synchrotron radiation comes from a region where the magnetic field is close to equipartition even though the magnetic field just behind the shock transition is a factor of 10 below the equipartition value $\left(\delta_{2} \approx 10^{-2}\right)$. In case $\mathrm{B}$, synchrotron emission is dominant and contributes $84 \%$ of the integrated total emission. The compression behind the shock is small and the magnetic field is hardly amplified, so even near $B_{\max }$ the synchrotron fraction of the total emission $(86 \%)$ is only slightly higher than the overall fraction. In case $\mathrm{C}$, the values of $\eta$ and $u_{A 1}$ are the same as in case A, and therefore the initial and final values of the magnetic field are the same. However, in case $\mathrm{C}$ the emission is dominated by IC scattering of external photons $(b=100)$, which are absent

in case A. Synchrotron emission now constitutes only $13 \%$ of the integrated total emission, but near $B_{\max }$ it contributes $59 \%$ of the total. Most of the synchrotron emission comes from a region where the magnetic field is close to equipartition - a larger fraction than in case A. This is a reflection of the fact that the ratio of synchrotron to total emission near $B_{\max }$ relative to that near $B_{2}$ is larger in case $\mathrm{C}$ because of the added cooling by IC scattering of the external photons.

\section{Applications}

To assess the relevance of cooling-induced magnetic field amplification to relativistic shock sources, one needs to address the following questions: What are the likely values of the normalized energy densities of the electrons/positrons and the magnetic field $\left(\epsilon_{e}\right.$ and $\epsilon_{B}$, respectively; see $\S 1$ ) in the emission region? What are the expected values of $\epsilon_{e}$ and $\epsilon_{B}$ immediately behind the shock transition (which can be written, in terms of the shock parameters defined in $\S 2$, as $\epsilon_{e 2}=\eta$ and $\left.\epsilon_{B 2}=\delta_{2} / 3\right)$ ? Is the shock radiative? If the shock is radiative but $\epsilon_{B 2}$ is lower than the observationally estimated value of $\epsilon_{B}$, can the compressional amplification of the magnetic field in the radiative zone make up the difference?

A strong relativistic shock $\left(u_{1} \gg 1, u_{A 1} \ll 1\right)$ with a transverse magnetic field satisfies $e_{2} \approx 2 u_{1}^{2} w_{1}$ and $B_{2}^{2} \approx 8 u_{1}^{2} B_{1}^{2}$ (see $\S 2$ ), implying that the ratio of the magnetic energy density to the total matter energy density is approximately constant across the shock and hence that $\epsilon_{B 2}$ can be expressed in terms of the enthalpy density $w_{1}\left(=\rho_{1} c^{2}\right.$ for a "cold" upstream medium) and the magnetic field amplitude $B_{1}$ ahead of the shock:

$$
\epsilon_{B 2} \approx \frac{B_{1}^{2}}{2 \pi \rho_{1} c^{2}}
$$

As a concrete example of how the above considerations can be applied, we again focus on GRBs and their afterglows. However, at the end of this section we comment briefly 
on AGNs. Starting with the $\gamma$-ray bursts themselves, we have already observed (see $\S 1$ ) that efficiency considerations imply that $\epsilon_{e}$ (in particular) and $\epsilon_{B}$ cannot be much smaller than 1 in the emission region. The value of $\epsilon_{B}$ may be constrained by observations through the requirement that the cooling time does not exceed the variability time scale $\delta t$. This translates into the condition that the Lorentz factor of an electron that cools on the time $\delta t$, $6 \pi m_{e} c / \sigma_{T} B^{2} \gamma \delta t\left(1+Y_{2}\right)$, must remain smaller than the characteristic synchrotron Lorentz factor, $\left(2 \pi m_{e} c \nu_{\mathrm{ob}} / \gamma e B\right)^{1 / 2}$, that corresponds to the observed frequency $\nu_{\mathrm{ob}}{ }^{\mathrm{W}}$ Following the same steps as in the derivation of equation (39) and again setting $\gamma_{12}=2$, we deduce

$$
\epsilon_{B}>8.3 \times 10^{-8}\left(1+Y_{2}\right)^{-4 / 3} E_{52}^{-1} \gamma_{2}^{16 / 3} t_{\mathrm{dur}, 1}^{5 / 3} \zeta_{-2}^{2 / 3}\left(\frac{h \nu_{\mathrm{ob}}}{100 \mathrm{keV}}\right)^{-2 / 3}
$$

(cf. Sari \& Piran 1997a). When the energy density of external photons is negligible, one has $Y_{2} \approx y$ (see eq. [33] with $b=0$ ), so that, if $y \gg 1$ (in which case $y \sim\left(\epsilon_{e 2} / \epsilon_{B 2}\right)^{1 / 2}$; see eqs. 21] and [22]), the constraint (43) on $\epsilon_{B}$ would be relaxed. However, $y \gg 1$ means that IC is dominant, with the observed synchrotron radiation being only a fraction $\sim 1 / y$ of the total radiated energy. The requirement of a reasonable radiative efficiency would then constrain $\epsilon_{B}$ to be not much smaller than $\epsilon_{e}$ (e.g., Piran 1999). The latter restriction would not arise if the observed $\gamma$-ray emission represented the SSC rather than the synchrotron component, but the synchrotron model appears to account quite well for the observed spectra (e.g., Lloyd \& Petrosian 2000), whereas an SSC interpretation could entail a prohibitively large energy input at the source."1]

As we have already noted, the prompt $\gamma$-ray emission in GRBs is believed to be the result of internal shocks within the flow. The relevant preshock field is then the relic magnetic field advected from the origin, and, to the extent that flux freezing is maintained,

\footnotetext{
${ }^{12}$ To simplify the discussion, we omit cosmological correction factors from the expressions presented in this paper.

${ }^{13}$ For example, in the model of Panaitescu and Mészáros (2000), the observed $\gamma$-ray spectrum is identified with a singly scattered SSC component. The characteristic thermal Lorentz factor of the emitting electrons in this picture is $\sim 30$, which implies that the peak synchrotron frequency is not very far from the optical regime. There should thus be an optical pulse that coincides with the prompt $\gamma$-ray emission, and the fact that such a component has not been detected (e.g., Akerlof et al. 2000; Williams et al. 2000) indicates that the Compton $y$ parameter is very large (Panaitescu and Mészáros 2000 estimate $y \gtrsim 5000$ ). Now, for the postulated thermal Lorentz factor, a typical seed photon undergoes two scatterings before the Klein-Nishina limit is reached. Since the emissivity ratios of the synchrotron, singly scattered SSC, and doubly scattered SSC components are $1: y: y^{2}$, it follows that the observed $\gamma$-ray pulse may constitute only a fraction $\sim 1 / y \ll 1$ of the total burst emission (and hence that the energy requirement on the source is a factor of $y$ larger).
} 
$\epsilon_{B 2} \propto B_{1}^{2} / \rho_{1}$ keeps the value that was imprinted on the ejecta at the source. $\square^{\mathbb{4}}$ Spruit et al. (2001) pointed out that, under these circumstances, values of $\epsilon_{B 2}$ as high as $\sim 0.1-1$ may be expected in the commonly invoked GRB models that envision large-scale, ordered magnetic fields actively driving the relativistic outflow by tapping the rotational energy of the central source. In particular, $\epsilon_{B 2}$ could well be $\sim 1$ in collimated (jet-like) outflows of this type. Spruit et al. further estimated that, if the field is "passive" (in that it does not drive the flow but is merely advected outward from the source), then $\epsilon_{B 2}$ would lie in the range $\sim 10^{-6}-10^{-3}$. In the latter case, compressional magnetic field amplification in the radiative zone could increase the fraction of radiation going into the synchrotron component (see Figs. 9 and 10). For this mechanism to operate, it is necessary that the radiative cooling time be much shorter than the dynamical time and that $\epsilon_{e}$ be $\sim 1$. The first requirement should in general be satisfied in internal shocks that produce GRBs (e.g., Piran 1999). The conditions under which $\epsilon_{e}$ could be close to 1 are somewhat uncertain, but there are several conceivable mechanisms for depositing a significant fraction of the postshock internal energy in the electron component even if the outflow consists predominantly of an electron-proton plasma (e.g., Bykov \& Mészáros 1996). It should be even easier to attain high values of $\epsilon_{e}$ if the plasma is dominated by $e^{ \pm}$pairs, a situation that in fact arises naturally in some GRB scenarios that involve magnetically driven outflows (e.g., Usov 1994; Mészáros \& Rees 1997).

The long-lived afterglow emission of GRBs is most likely associated with the forward shock that propagates into the ambient medium. We have already noted in $\S 1$ that comparatively high values of $\epsilon_{e}(\sim 0.1-1)$ and $\epsilon_{B}(\sim 0.01-0.1)$ have been directly inferred from observations of the GRB 970508 afterglow. These parameters have so far been less well constrained in other sources, but in a recent modeling of four other afterglows (Panaitescu $\&$ Kumar 2001) values in the range $10^{-2} \lesssim \epsilon_{e} \lesssim 10^{-1}$ and $10^{-6} \lesssim \epsilon_{B} \lesssim 10^{-1}$ have been inferred. It is also worth noting in this connection the suggestion by Galama et al. (1999) that comparatively low values of $\epsilon_{B}$ may characterize afterglow sources that exhibit weak radio emission (attributed by them to low values of the characteristic frequency and peak

\footnotetext{
${ }^{14}$ Note in this connection that equation (42) shows quite generally that $\epsilon_{B 2}$ will remain constant with time if the medium into which the shock propagates is either a supersonic outflow with a predominantly transverse magnetic field that is frozen into the matter (in which case both $B_{1}$ and $\rho_{1}^{1 / 2}$ scale inversely with distance from the center) or else is uniform (in which case the values of $B_{1}$ and $\rho_{1}$ are fixed). The former case applies to internal shocks and to the reverse shock (which propagate in the ejecta) as well as to a forward shock that propagates in a magnetized stellar-wind environment. The latter case corresponds to a forward shock in a uniform ISM. This shows that the predictions of conventional emission models of GRBs and their afterglows, which simply postulate a constant postshock value of $\epsilon_{B}$, will by and large continue to apply when this parameter is calculated self-consistently.
} 
flux of the synchrotron spectrum, both of which scale as $\epsilon_{B}^{1 / 2}$ ). The expected values of $\epsilon_{e 2}$ and $\epsilon_{B 2}$ in GRB afterglows depend on the GRB environment (which directly affects such factors as the pair content, the electron/positron acceleration efficiency, and the degree of magnetization in the shocked gas). The uniform-density model fittings of the GRB 970508 afterglow (Wijers \& Galama 1999; Granot, Piran, \& Sari 1999) yielded upstream densities in the range $\sim 0.03-3 \mathrm{~cm}^{-3}$, which are typical of a diffuse ISM. In the case of GRB 980329, a preshock density $\sim 10^{3} \mathrm{~cm}^{-3}$, which is typical of a molecular cloud, has been deduced (Lamb et al. 1999). In the Galactic ISM, the inferred magnitude of the Alfvén speed does not vary strongly from diffuse to dense environments and corresponds to values of $\epsilon_{B 2}$ in the range $\sim 3 \times 10^{-11}-3 \times 10^{-10}$. If these values are also typical of the host galaxies of GRBs, then the synchrotron emission from their associated afterglows would in most cases be too weak to be detected. However, all the afterglow sources observed so far correspond to "long" bursts $\left(t_{\text {dur }} \gtrsim 2 \mathrm{~s}\right.$ ), for which a plausible origin is the collapse of a massive (e.g., Wolf-Rayet) star (e.g., Woosley 2000; Mészáros 2000). In this case, the medium into which the forward shock expands could correspond to the preburst wind from the central star (e.g., Chevalier \& Li 2000; Ramirez-Ruiz et al. 2001). It has been suggested (e.g., Biermann \& Cassinelli 1993) that winds from Wolf-Rayet stars are magnetically driven and have spatially constant Alfvén speeds corresponding to values of $\epsilon_{B 2}$ that can be as high as $\sim 10^{-4}$. If such winds constituted the environment into which the forward shock expands, then they could account for afterglows with the lowest values of $\epsilon_{B}$ inferred to date even if there were no additional magnetic field amplification beyond that induced by the passage of the ambient field through the shock transition. Radiative cooling effects could increase the magnitude of $\epsilon_{B}$ in the afterglow emission region above its value immediately behind the forward shock. The cooling time behind the forward shock is typically shorter than the dynamical time during the early phase of the afterglow, but subsequently this inequality is reversed. For a shock propagating into a uniform-density ISM, the time of transition from radiative to adiabatic evolution was calculated by Sari et al. (1998) and is given by

$$
t_{\mathrm{rad} \rightarrow \mathrm{ad}}=4.6 \epsilon_{B 2}^{7 / 5} \epsilon_{e 2}^{7 / 5}\left(1+Y_{2}\right)^{7 / 5} E_{52}^{4 / 5} \gamma_{0,2}^{-4 / 5} n_{1}^{3 / 5} \text { days (ISM), }
$$

where $\gamma_{0}$ is the initial Lorentz factor of the ejecta. In the absence of a strong coupling between protons and electrons behind the shock transition, $\epsilon_{e 2} \approx 1$ is required for the shock to be radiative. Equation (44) indicates that, even if this condition is satisfied, $\left(1+Y_{2}\right) \epsilon_{B 2}$ cannot be much smaller than 1 if the radiative phase is to last through a significant fraction of the early evolution of a typical afterglow (i.e., a few days or so). Taking $\epsilon_{B 2} \approx 10^{-10}$ as a representative ISM value, we infer $\left(1+Y_{2}\right) \epsilon_{B 2} \sim\left(\epsilon_{e 2} \epsilon_{B 2}\right)^{1 / 2} \sim 10^{-5}$, implying that $t_{\mathrm{rad} \rightarrow \text { ad }}$ is only a few seconds! In fact, in this case, even if the radiative phase lasted longer, the expected magnetic field amplification in the radiative zone would not be sufficient to raise $\epsilon_{B}$ to a detectable level (see $\left.\$ 3.3\right)$. 
In the case of a stellar-wind environment, the ambient density scales with distance $r$ from the source as $\rho(r)=A r^{-2}$, where the constant $A \equiv \dot{M} / 4 \pi v_{w}$ is defined in terms of the wind mass-loss rate $\dot{M}$ and speed $v_{w}$. We are interested in the evolutionary phase during which the forward shock has already decelerated significantly but is still relativistic. Assuming that the mass is concentrated in a thin, spherical shell of radius $R$ and Lorentz factor $\gamma$, we can write $\gamma(R)=M / m(R)$, where $M\left(=E / \gamma_{0} c^{2}\right)$ is the initial mass of the ejecta and $m(R)$ is the rest mass of the swept-up matter (Blandford \& McKee 1976; Katz \& Piran 1997). In our case $m(R)=4 \pi A R$ and therefore $\gamma(R)=L / R$, where $L=M / 4 \pi A$ is the distance from the center where the flow becomes nonrelativistic. Using $t=R / 4 \gamma^{2} c$, we obtain

$$
\begin{aligned}
& \gamma=\left(\frac{E}{16 \pi A \gamma_{0} c^{3} t}\right)^{1 / 3}, \\
& R=\left(\frac{E^{2} t}{4 \pi^{2} A^{2} \gamma_{0}^{2} c^{3}}\right)^{1 / 3} .
\end{aligned}
$$

The Lorentz factor of an electron that cools on a time scale $t$ is given by

$$
\gamma_{c}=\frac{6 \pi m_{e} c}{\sigma_{T}\left(1+Y_{2}\right) \gamma B^{2} t},
$$

whereas the typical Lorentz factor of the electrons can be approximated by

$$
\gamma_{m} \approx 610 \epsilon_{e 2} \gamma
$$

(e.g., Sari et al. 1998). Assuming that the preshock field $B_{1}=B_{2} / \gamma_{1}$ scales as $1 / R$ (e.g., Biermann \& Cassinelli 1993), we find $\gamma_{c}(t) \propto t^{2 / 3}$, so the ratio of the radiative cooling time to the dynamical time $\left(t_{\mathrm{rad}} / t_{\mathrm{dyn}}=\gamma_{c} / \gamma_{m}\right)$ is $\propto t$. This shows explicitly that at early times $t_{\mathrm{rad}}<t_{\mathrm{dyn}}$ and the evolution is radiative. The transition from the radiative to the adiabatic phase occurs approximately when $\gamma_{c} / \gamma_{m}$ increases to 1 . Using $B^{2} / 8 \pi=\epsilon_{B} e$ and $e=4 \gamma^{2} \rho c^{2}$, we obtain

$$
t_{\mathrm{rad} \rightarrow \mathrm{ad}}=28.8 \epsilon_{B 2} \epsilon_{e 2}\left(1+Y_{2}\right) A_{*} \text { days (Wind), }
$$

where $A_{*} \equiv A /\left(5 \times 10^{11} \mathrm{~g} \mathrm{~cm}^{-1}\right)$ corresponds to a stellar wind characterized by a speed of $10^{8} \mathrm{~cm} \mathrm{~s}^{-1}$ and a mass loss rate of $10^{-5} M_{\odot} \mathrm{yr}^{-1}$ (Chevalier \& Li 2000). As can be seen from a comparison of equations (44) and (49), the radiative phase of the forward shock will typically last longer in a wind environment than in a uniform-density medium, which can be attributed (Chevalier \& Li 2000) to the higher ambient density encountered at early times by a shock that propagates into a wind. Using equations (21) and (22) to approximate $\epsilon_{e 2} \epsilon_{B 2}\left(1+Y_{2}\right) \lesssim\left(\epsilon_{B 2} / 2\right)^{1 / 2}$ and setting $\epsilon_{B 2} \approx 10^{-4}$ (the expected value in a strongly magnetized Wolf-Rayet outflow), we infer from equation (49) that $t_{\mathrm{rad} \rightarrow \mathrm{ad}} \lesssim 5 A_{*} \mathrm{hr}$. During 
the radiative phase, a typical electron could radiate up to $\sim 10 \%$ of its postshock random energy in a region with $\epsilon_{B} \gtrsim 10^{-2}$ (see Fig. 9). However, the estimated value of $t_{\mathrm{rad} \rightarrow \text { ad }}$ is too small for the radiative field-amplification mechanism to apply to the observations of a source like GRB 970508, in which values of $\epsilon_{B} \gtrsim 10^{-2}$ have been inferred over substantially longer time scales. Furthermore, even if the model were applicable, this mechanism could not account for a value of $\epsilon_{B}$ as high as 0.1 (the best-fit value for a stellar-wind model; Chevalier \& Li 2000) if $\epsilon_{B 2}$ is not larger than $\sim 10^{-4}$.

Another potential source of detectable emission in GRB sources is the reverse shock, which runs into the ejecta shell when the latter starts to be decelerated by the inertia of the swept-up ambient gas. In fact, a shock of this type is believed to be the source of the prompt optical emission (or "optical flash") that was observed in GRB 990123 (Akerlof et. al. 1999; Sari \& Piran 1999a,b; Mészáros \& Rees 1999) as well as of a rapidly decaying "radio flare" detected about a day after the burst in this source (and evidently also present in GRB 990123; Kulkarni et al. 1999). So far, however, these observations have not provided strong constraints on the parameters $\epsilon_{e}$ and $\epsilon_{B}$ in the emission region. It may, however, be expected that, since the reverse shock propagates into the ejecta, it is characterized by values of $\epsilon_{e 2}$ and $\epsilon_{B 2}$ that are similar to those in internal shocks.

GRB 990123 was a comparatively long $\gamma$-ray burst: such sources likely possess a relatively thick ejecta shell (of thickness $\Delta$ as measured by a stationary local observer), and their prompt optical emission should peak at $t_{\text {peak }} \approx \Delta / c$, the shock crossing time of the shell (which is approximately equal to the duration of the burst), by which time the reverse shock would be relativistic (e.g., Sari \& Piran 1999b). This is a natural time scale to use in a comparison with the cooling time for determining whether the shock is radiative. We can repeat the procedure applied above to the forward shock by comparing the characteristic Lorentz factors $\gamma_{m}$ and $\gamma_{c}$, except that in this case we substitute for $\gamma$ in equation (47) for $\gamma_{c}$, by the Lorentz factor $\gamma_{3}$ of the shocked shell material, which is given in terms of the Lorentz factor $\gamma_{\mathrm{sh}}$ of the un-shocked portion of the ejecta shell (both measured in the stationary frame) by

$$
\gamma_{3}=\gamma_{\mathrm{sh}}^{1 / 2} f^{1 / 4} / \sqrt{2}
$$

(e.g., Sari \& Piran 1995), where

$$
f=\frac{E}{4 \pi \gamma_{\mathrm{sh}}^{2} R_{\Delta}^{2} \Delta n_{p 1} m_{p} c^{2}}
$$

is the ratio of the proper mass densities in the shell and the ambient medium (assumed to be dominated by protons) and $R_{\Delta}=\gamma_{3}^{2} \Delta$ is the radius where the reverse shock crosses the shell. Similarly, we replace $\gamma$ in equation (48) for $\gamma_{m}$ by the relative Lorentz factor between the shocked and the unshocked shell material, $\bar{\gamma}_{3}=\gamma_{3} / \sqrt{f}$. 
In the case of a uniform ambient medium, the condition $\gamma_{c}\left(t_{\text {peak }}\right) / \gamma_{m}\left(t_{\text {peak }}\right) \leq 1$ implies

$$
\epsilon_{e 2} \epsilon_{B 2}\left(1+Y_{2}\right) \geq 0.12 E_{52}^{-1 / 4} n_{p 1}^{-3 / 4}\left(t_{\text {peak }} / 50 \mathrm{~s}\right)^{-1 / 4}\left(\gamma_{\mathrm{sh}} / 200\right)^{-1}
$$

where we have normalized $t_{\text {peak }}$ and $\gamma_{\text {sh }}$ by their estimated values in GRB 990123 [Sari \& Piran 1999a). 四 Assuming $\epsilon_{e 2} \sim 1 \gg \epsilon_{B 2}$ [which implies, in the absence of an external radiation field, $\left.\epsilon_{e 2} \epsilon_{B 2}\left(1+Y_{2}\right) \lesssim\left(\epsilon_{B 2} / 2\right)^{1 / 2}\right]$, we obtain a lower bound on $\epsilon_{B 2}$,

$$
\epsilon_{B 2} \gtrsim 0.03 E_{52}^{-1 / 2} n_{p 1}^{-3 / 2}\left(t_{\text {peak }} / 50 \mathrm{~s}\right)^{-1 / 2}\left(\gamma_{\mathrm{sh}} / 200\right)^{-2} .
$$

The inequality (53) could in principle be satisfied if the GRB outflow is driven magnetically, in which case the ejecta could be characterized by $\epsilon_{B 1} \sim \epsilon_{B 2} \sim 1$. However, even if $\epsilon_{B 2}$ is near its estimated lower bound, compressional amplification could boost the magnetic field to equipartition values in the radiative zone (see Figs. 8 and 9). The corresponding condition for a stellar wind environment (assuming strong hydrogen depletion as appropriate for a Wolf-Rayet progenitor) is

$$
\epsilon_{e 2} \epsilon_{B 2}\left(1+Y_{2}\right) \geq 9.6 \times 10^{-6} E_{52}^{1 / 2} A_{*}^{-3 / 2}\left(t_{\text {peak }} / 50 \mathrm{~s}\right)^{1 / 2}\left(\gamma_{\mathrm{sh}} / 200\right)^{-1}
$$

(Chevalier \& Li 2000). For $\epsilon_{e 2} \sim 1 \gg \epsilon_{B 2}$ and a negligible external radiation field, equation (54) implies

$$
\epsilon_{B 2} \gtrsim 1.8 \times 10^{-10} E_{52} A_{*}^{-3}\left(t_{\text {peak }} / 50 \mathrm{~s}\right)\left(\gamma_{\mathrm{sh}} / 200\right)^{-2},
$$

which demonstrates that the reverse shock is always radiative in this case for plausible parameter values. It is worth noting, however, that if the reverse shock is radiative at the time that it crosses the shell, then it will rapidly cool and become invisible at later times (e.g., Sari \& Piran 1999a). Since this was not the case for the optical flash in GRB 990123, we can conclude, on the basis of equations (54) and (52), that the outflow in this source does not expand into a wind (as was already inferred by Chevalier \& Li 2000) and that the value of $\epsilon_{e 2} \epsilon_{B 2}$ behind the reverse shock is $<0.1$.

We can summarize our discussion of GRB sources as follows. The field amplification mechanism discussed in this paper could in principle operate in shocks that are driven into the ejecta: either the internal shocks that give rise to the prompt $\gamma$-ray emission,

\footnotetext{
${ }^{15}$ The condition $\gamma_{c}=\gamma_{m}$ is probably a bit conservative. For one thing, as we have demonstrated in $\S 3$, the cooling induces compression in the radiative layer, which has the effect of enhancing the radiative losses. Furthermore, the radiative efficiency decreases only gradually after $\gamma_{c}$ comes to exceed $\gamma_{m}$ : in fact, the fraction of the internal electron/positron energy that is radiated by the synchrotron process after that time is given by $\left(\gamma_{m} / \gamma_{c}\right)^{p-2}$, where $p$ (which has a canonical value of 2.5) is the power-law index of the radiating particle distribution (e.g., Moderski, Sikora, \& Bulik 2000). Thus, the right-hand sides of the inequalities (52) $-(55)$ are probably only upper limits.
} 
or the reverse shock that produces the optical flash and radio flare emission. Except for reverse shocks in sources with a uniform ambient medium in the case when the condition (52) is not satisfied, these shocks will be radiative if most of the energy dissipated in the shock transition is deposited into the electron/positron component. Compressional field amplification in the radiative zone of these shocks could enable a significant fraction $(\sim 0.1-0.3)$ of the radiated energy to be emitted from regions where the field is substantially stronger (by up to $\sim 1-2$ orders of magnitude in $\epsilon_{B}$ ) than immediately behind the shock transition. If the preshock magnetization is comparatively weak $\left(\epsilon_{B 1} \ll 1\right)$, the radiative amplification could significantly enhance the synchrotron emission and may in certain cases help to bring it above the detection limit. If the ejecta is already moderately strongly magnetized $\left(\epsilon_{B 1} \gtrsim 10^{-2}\right)$, this process could increase the field in the emission region to equipartition values.

We have also concluded that the compressional amplification mechanism will not significantly affect the afterglow emission of GRB sources, both because the forward shock typically undergoes a transition to adiabatic evolution early on, and because, even during the radiative phase, the expected amplification falls short of the required enhancement for standard ISM environments. We noted that magnetized stellar winds from Wolf-Rayet stars could provide an adequate environment in which afterglows with values of $\epsilon_{B}$ that lie near the low end of the observationally inferred range would be produced in adiabatic shocks without requiring further field amplification. However, the comparatively high $\left(z 10^{-2}\right)$ values of $\epsilon_{B}$ indicated in a source like GRB 970508 cannot be accounted for by the basic shock model and typical ISM parameters. We do not pursue this topic further in this paper, but we note that several suggestions that bear on the need to have high values of $\epsilon_{B}$ and $\epsilon_{e}$ in such shocks have already been made in the literature (e.g., Medvedev \& Loeb 1999; Smolsky \& Usov 2000; Thompson \& Madau 2000; Lyutikov \& Blackman 2001; König1 \& Granot 2001).

The observational data on spectral flares in AGNs are not as extensive as in GRB sources, and, correspondingly, their theoretical study has so far been less developed. However, as we noted in $\S 1$, the blazar flares have been similarly interpreted in terms of nonthermal emission from relativistic, and likely highly collimated, outflows from the vicinity of a central compact object..$^{m}$ In particular, the variable emission has been modeled in terms of internal shocks in a magnetized relativistic outflow, possibly formed from

\footnotetext{
${ }^{16}$ One key difference between the high-energy flares in AGNs and GRB sources is in their observed duration, which is of the order of hours in blazars (e.g., Wehrle et al. 1998; Maraschi et al. 1999) as compared with seconds in GRBs. A related difference is in the inferred bulk Lorentz factor of the outflow: $\lesssim 10$ in blazars vs. $\gtrsim 10^{2}$ in GRBs.
} 
overtaking collisions of disturbances ("shells") in the flow (e.g., Marscher \& Gear 1995; Romanova \& Lovelace 1997; Sikora et al. 1997; Levinson 1998, 1999). In these models, the high-energy emission is attributed to an SSC or an ERC component. The quasi-isotropic external radiation field invoked in the ERC interpretations can arise from the nuclear accretion disk (e.g., Dermer \& Schlikeiser 1994), or, perhaps more likely, from scattering by the broad emission-line region (BELR) "clouds" of the nuclear continuum radiation (e.g., Sikora, Begelman, \& Rees 1994; Blandford \& Levinson 1995) or of the beamed jet emission itself (Ghisellini \& Madau 1996), as well as from the near-infrared emission of warm dust outside the BELR (Błażejowski et al. 2000). As we discussed in $\$ 3.3$, if the emission from a radiative shock is dominated by IC scattering of external photons, then the synchrotron radiation component of the shock will have a relatively stronger contribution from regions of high values of $B / B_{\text {eq }}$ (see Fig. 10). M Most of the blazar emission models considered to date have, however, taken $\gamma_{m}$ to lie below $\gamma_{c}$ (e.g., Maraschi, Ghisellini, \& Celotti 1992; Sikora et al. 1994; but see Sikora et al. 2001) and thus correspond to nonradiative shocks. It is, nonetheless, likely that the cooling times could be quite short in these sources, as suggested, for example, by very rapid flares in blazars like 3C 279 (e.g., Wehrle et al. 1998), Mrk 421 (e.g., Maraschi et al. 1999), and AO 0235+164 (e.g., Kraus et al. 1999). Furthermore, there are indications that AGN jets may contain a significant, or even dominant, electron/positron component (e.g., Reynolds et al. 1996; Wardle et al. 1998; Sikora \& Madejski 2000), which would naturally lead to high values of $\epsilon_{e}$ in the shocks. Given that the outflows are likely magnetically driven (e.g., Blandford \& Znajek 1977; Blandford \& Payne 1982), comparatively high values of $\epsilon_{B}$ may also be expected. It is thus conceivable that at least some of the synchrotron emission from these sources originates in radiative shocks in which the magnetic field is amplified by cooling-induced compression.

\footnotetext{
${ }^{17}$ The emission characteristics of GRB sources might also be affected by the interaction of the outflow with an external radiation field. For example, Lazatti et al. (2000) considered the possibility that the prompt $\gamma$-ray emission arises in the course of the Compton-drag deceleration of a relativistic outflow as it propagates through the debris of a progenitor massive star; in this scenario, magnetic fields play no role in the emission process. On larger scales, Madau, Blandford, \& Rees (2000) proposed that backscattering of the GRB radiation by the comparatively dense massive-star environment could lead to a delayed $\mathrm{MeV}$ emission, again through the Compton-drag effect on the bulk flow. In the latter case, ERC emission by a "hot" (i.e., relativistic) electron/positron component of the flow could in principle also contribute to the observed radiation (cf. the analogous proposal for blazars by Ghisellini \& Madau 1996). So far, however, there has been no discussion in the literature of the possible appearance of an ERC emission component in GRB sources.
} 


\section{Conclusions}

Gamma-ray bursts and their afterglows, as well as energetic flares from blazars and miniquasars, are commonly interpreted in terms of shocks in a relativistic outflow from a compact object that emit nonthermal (synchrotron and inverse Compton) radiation. In this paper we have investigated the structure of radiative shocks in such sources. A shock is "radiative" if the emission mechanism can tap into the bulk of the postshock internal energy and if the radiative cooling time behind the shock transition is shorter than the relevant dynamical time (the characteristic time for adiabatic energy losses). Such a shock is characterized by a radiative zone of finite width where most of the energy dissipated in the shock transition front is radiated away. The structure of radiative shocks has been previously studied in the nonrelativistic regime in the context of the interstellar medium, where the dominant emission processes are thermal. One particularly interesting finding of these studies has been the strong effect that such a shock could have on the normal component of a magnetic field that is frozen into the gas, and, in turn, the potentially important role that the field could play in limiting the compression in the shock. When the shock is nonradiative, the compression $\left(n_{2} / n_{1}\right)$ and resultant magnetic field amplification $\left(B_{2} / B_{1}=n_{2} / n_{1}\right)$ have a value of at most a few in the nonrelativistic regime (generalizing to a few times the shock Lorentz factor $\gamma_{1}$ in the relativistic case, with $n$ and $B$ measured in the fluid rest frame). Efficient cooling can lead to a strong enhancement of the density and the field amplitude in the radiative zone, but when the magnetic pressure attains equipartition with the thermal pressure, further compression is inhibited and subsequent cooling proceeds at nearly constant density. We have shown that, when synchrotron radiation dominates the cooling, the interplay between the cooling and the compression becomes more pronounced on account of the feedback effect between the field amplification and the emission process: a stronger magnetic field increases the emissivity, which in turn induces a larger compression that further amplifies the field.

The cooling-induced compressional field amplification may have potentially significant consequences in sources that harbor radiative shocks: if the preshock magnetic field is low, this mechanism could in some cases increase it to a level where the synchrotron radiation becomes detectable, and if the preshock field is already moderately strong (so that it is within an order of magnitude of equipartition in the shocked but uncooled gas), this process could result in a considerable fraction $(z 0.1)$ of the radiation being emitted from regions where $B \approx B_{\text {eq }}$. When the shock is radiative but the field immediately behind the shock transition is below equipartition, the initial cooling would be dominated by the inverse-Compton process: either synchrotron self-Compton (SSC) or external-radiation Compton (ERC), depending on which seed-photon energy density is higher in the shock frame. In this case, a larger fraction of the synchrotron emission comes from regions with a 
stronger magnetic field if ERC dominates SSC.

For standard scenarios of GRB outflows and their environments, this field amplification mechanism may be relevant to internal shocks that produce the prompt $\gamma$-ray emission and possibly also to the reverse shock responsible for the optical "flash" and radio "flare" in sources like GRB 990123. It will likely not be of much relevance to the forward shock that gives rise to the GRB afterglow, although we pointed out that if the shock expands into a magnetized wind from a Wolf-Rayet star then no further field amplification would be required to account for the lowest $B / B_{\text {eq }}$ values that have so far been inferred from afterglow observations. We also suggested that radiative shocks in which this mechanism operates could occur in blazars.

We are grateful to Tsvi Piran for many valuable conversations. JG acknowledges a Priscilla and Steven Kersten Fellowship at the University of Chicago and thanks the Department of Astronomy and Astrophysics at the University of Chicago for its hospitality. AK acknowledges a Forchheimer Fellowship and thanks the Racah Institute at the Hebrew University for hospitality. This research was supported in part by the US-Israel BSF grant BSF-9800225 (JG) and by NASA grant NAG 5-9063 (AK).

\section{REFERENCES}

Akerlof, C., et al. 1999, Nature, 398, 400 2000, ApJ, 532, L25

Angel, J. R. P., \& Stockman, H. S. 1980, ARA\&A, 18, 321

Biermann, P. I., \& Cassinelli, J. P. 1993, A\&A, 277, 691

Blandford, R. D., \& Levinson, A. 1995, ApJ, 441, 79

Blandford, R. D. \& McKee, C. F. 1976, Phys. Fluids, 19, 1130

Blandford, R. D., \& Payne, D. G. 1982, MNRAS, 199, 883

Blandford, R. D., \& Znajek, R. L. 1977, MNRAS, 179, 433

Błażejowski, M., Sikora, M., Moderski, M., \& Madejski, G. 2000, ApJ, 545, 107

Bower, G. C., \& Backer, D. C. 1998, ApJ, 507, L117 
Bykov, A. M., \& Mészáros, P. 1996, ApJ, 461, L37

Chevalier, R.A., \& Li, Z.Y., 2000, ApJ, 536, 195

Cohen, E., \& Piran, T. 1999, ApJ, 518, 346

Cohen, E., Piran, T. \& Sari, R. 1998, ApJ, 509, 717

Covino, S., et al. 1999, A\&A, 348, L1

Dermer, C. D., \& Schlikeiser, R. 1994, ApJS, 90, 945

Draine, B. T., \& McKee, C. F. 1993, ARA\&A, 31, 373

Fenimore, E., et al. 1996, ApJ, 473, 998

Galama, T. J., et al. 1999, Nature, 398, 394

Ghisellini, G., \& Madau, P. 1996, MNRAS, 280, 67

Granot, J., Piran, T. \& Sari, R. 1999, ApJ, 527, 236 2000, ApJ, 534, L163

Hollenbach, D. J., \& McKee, C. F. 1979, ApJS, 41, 555

Kaiser, C. R., Sunyaev, R., \& Spruit, H. C. 2000, A\&A, 356, 975

Katz, J. I., \& Piran, T. 1997, ApJ, 490, 772

Königl, A. 1980, Phys. Fluids, 23, 1083

Königl, A., \& Granot, J. 2001, in preparation

Kraus, A., et al. 1999, A\&A, 344, 807

Kulkarni, S. R., et al. 1999, ApJ, 522, L97

Lamb, D. Q., Castander, F. J., \& Reichart, D. E. 1999, A\&AS, 138, 479

Lazzati, D., Ghisellini, G., Celotti, A., \& Rees, M. J. 2000, ApJ, 529, L17

Levinson, A. 1998, ApJ, 507, 145 1999, ApJ, 522, 93

Lloyd, N. M., \& Petrosian, V. 2000, ApJ, 543, 722 
Lyutikov, M., \& Blackman E. G. 2001, MNRAS, 321, 177

Madau, P., Blandford, R. D., \& Rees, M. J. 2000, ApJ, 541, 712

Maraschi, L., et al. 1999, ApJ, 526, L81

Maraschi, L., Ghisellini, G., \& Celotti, A. 1992, ApJ, 397, L5

Marscher, A. P., \& Gear, W. K. 1995, ApJ, 298, 114

Medvedev, M. V. 2000, ApJ, 540, 704

Medvedev, M. V., \& Loeb, A. 1999, ApJ, 526, 697

Melatos, A., \& Melrose, D. B. 1996, MNRAS, 279, 1168

Mészáros, P. 2000, Nucl. Phys. Proc. Suppl. 80, 63

Mészáros, P., \& Rees, M. J. 1997, ApJ, 482, L29 1999, MNRAS, 306, L39

Mirabel, I. F., \& Rodríguez, L. F. 1999, ARA\&A, 37, 409

Moderski, R., Sikora, M., \& Bulik, T. 2000, ApJ, 529, 151

Panaitescu, A., \& Kumar, P. 2001, ApJ, in press astro-ph/0010257)

Panaitescu, A., \& Mészáros, P. 2000, ApJ, 544, L17

Piran, T. 1999, Phys. Rep., 314, 575 .2000, Phys. Rep., 333, 529

Ramirez-Ruiz, E., Dray, L. M., Madau, P., \& Tout, C. A. 2001, MNRAS, in press (astro-ph/0012396)

Readhead, A. C. S. 1994, ApJ, 426, 51

Rees, M. J. 1978, MNRAS, 184, P61

Reynolds, C. S., Fabian, A. C., Celotti, A., \& Rees, M. J. 1996, MNRAS, 283, 873

Rol, E., et al. 2000, ApJ, 544, 707

Romanova, M. M., \& Lovelace, R. V. E. 1997, ApJ, 475, 97 
Rybicki, G.B. \& Lightman, A.P. 1979, Radiative Processes in Astrophysics (New York: Wiley-Interscience)

Sari, R., Narayan, R. \& Piran, T. 1996, ApJ, 473, 204

Sari, R. \& Piran, T. 1995, ApJ, 455, L143

. 1997, MNRAS, 287, 110 . 1997, ApJ, 485, 270 .1999a, ApJ, 517, L109 . 1999b, ApJ, 520, 641

Sari, R., Piran, T. \& Narayan, R. 1998, ApJ, 497, L17

Sikora, M, Begelman, M. C., \& Rees, M. J. 1994, ApJ, 421, 153

Sikora, M., Błażejowski, M., Begelman, M. C., \& Moderski, R. 2001, ApJ, in press (astro-ph/0102091)

Sikora, M., \& Madejski, G. 2000, ApJ, 534, 109

Sikora, M., Madejski, G., Moderski, R., \& Poutanen, J. 1997, ApJ, 484, 108

Smolsky, M. V., \& Usov, V. V. 2000, ApJ, 531, 764

Spruit, H.C., Daigne, F. \& Drenkhahn, G. 2001, A\&A, 369, 694

Thompson, C., \& Madau, P. 2000, ApJ, 538, 105

Ulrich, M.-H., Maraschi, L., \& Urry, C. M. 1997, ARA\&A, 35, 445

Usov, V. V. 1994, MNRAS, 267, 1035

Wardle, J. F. C., Homan, D. C., Ojha, R., \& Roberts, D. H. 1998, Nature, 395, 457

Wehrle, A. E., et al. 1998, ApJ, 497, 178

Wijers, R. A. M. J., et al. 1999, ApJ, 523, L33

Wijers, R. A. M. J., \& Galama, T. J. 1999, ApJ, 523, 177

Williams, G. G., et al. 2000, in AIP Conf. Proc. 526, 5th Huntsville Symp. on Gamma-Ray Bursts, ed. R. M. Kippen, R. S. Mallozzi, \& G. J. Fishman (New York: AIP), 250 
Woosley, S. E. 2000, in Fifth Huntsville Conference on Gamma-Ray Bursts, ed. R. M. Kippen, R.S. Mallozzi, \& V. Connaughton (New York: AIP), 555

Zhang, B., \& Mészáros, P. 2001, ApJ, in press astro-ph/0103229 


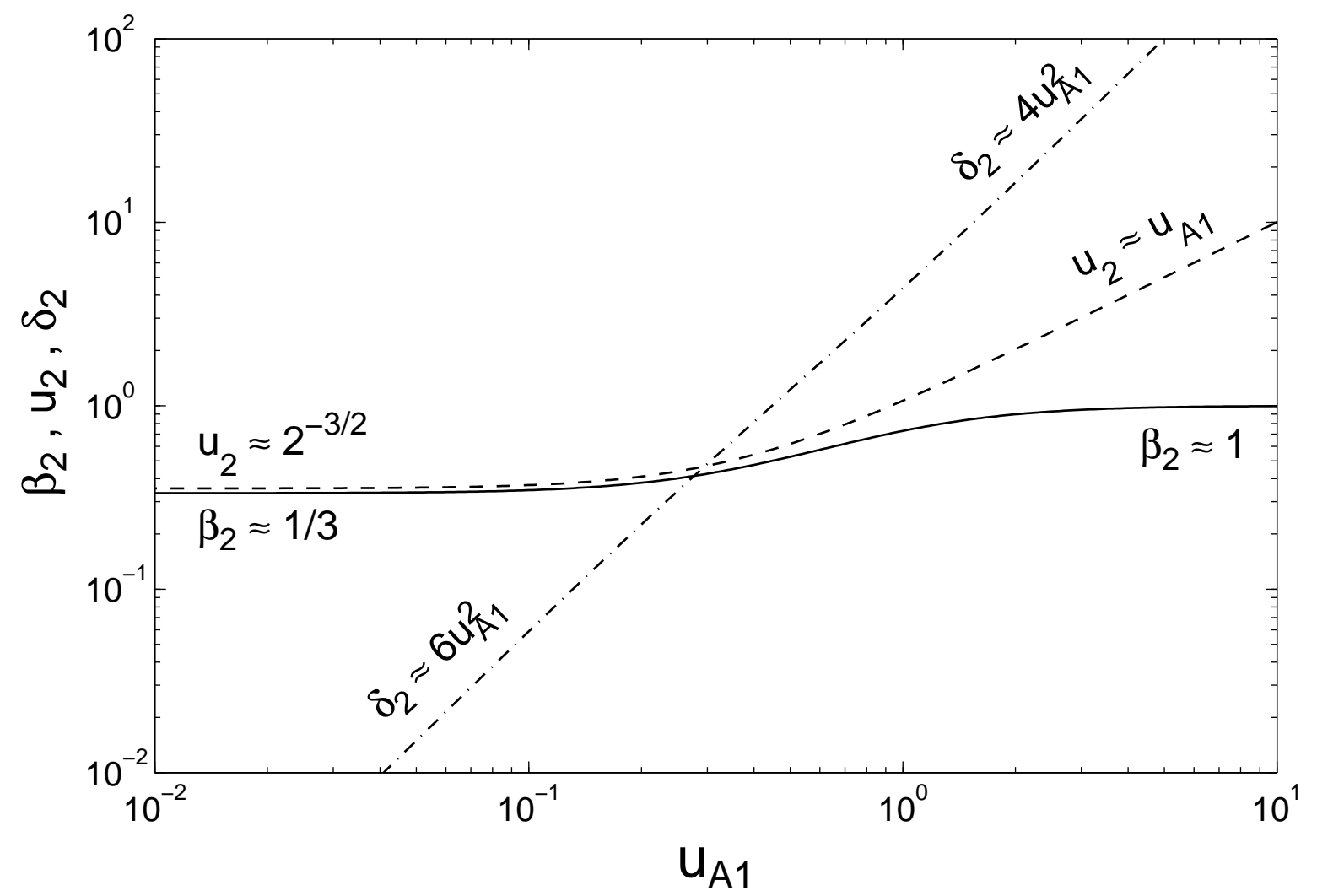

Fig. 1. - The speed ( $\beta_{2}$, solid line), proper speed $\left(u_{2}\right.$, dashed line), and magnetic-to-thermal pressure ratio $\left(\delta_{2}\right.$, dash-dotted line) immediately behind the shock transition, as a function of the proper Alfvén speed $u_{A 1}$. The asymptotic values for $u_{A 1}$ much larger or much smaller than 1 are given in the figure. In order for a shock wave to exist, $u_{A 1}$ must be smaller than the upstream speed $u_{1}$. 


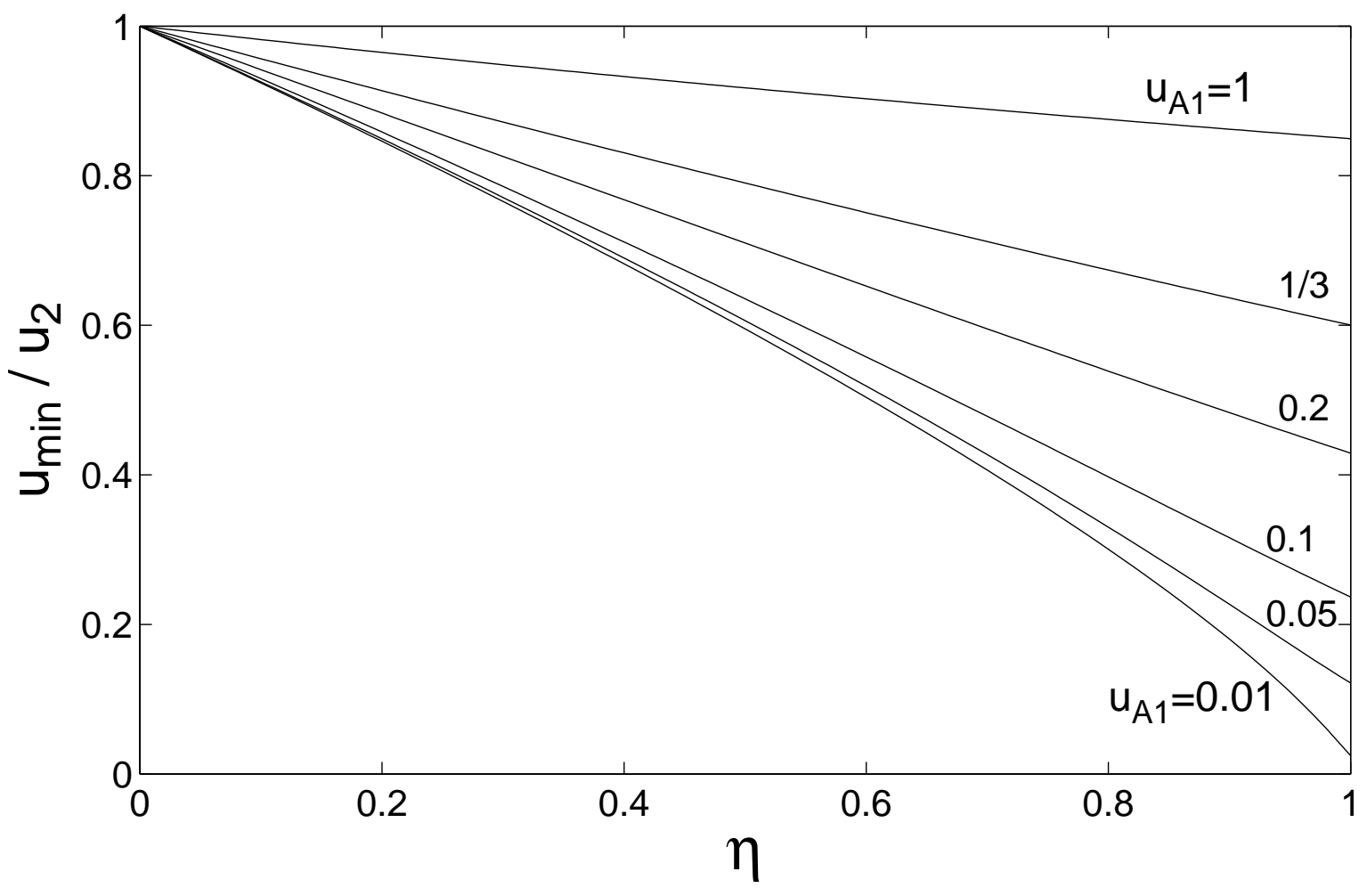

Fig. 2.- The asymptotic proper speed $u_{\text {min }}$ (in units of $u_{2}$ ) as a function of $\eta$ for several values of $u_{A 1}$. 


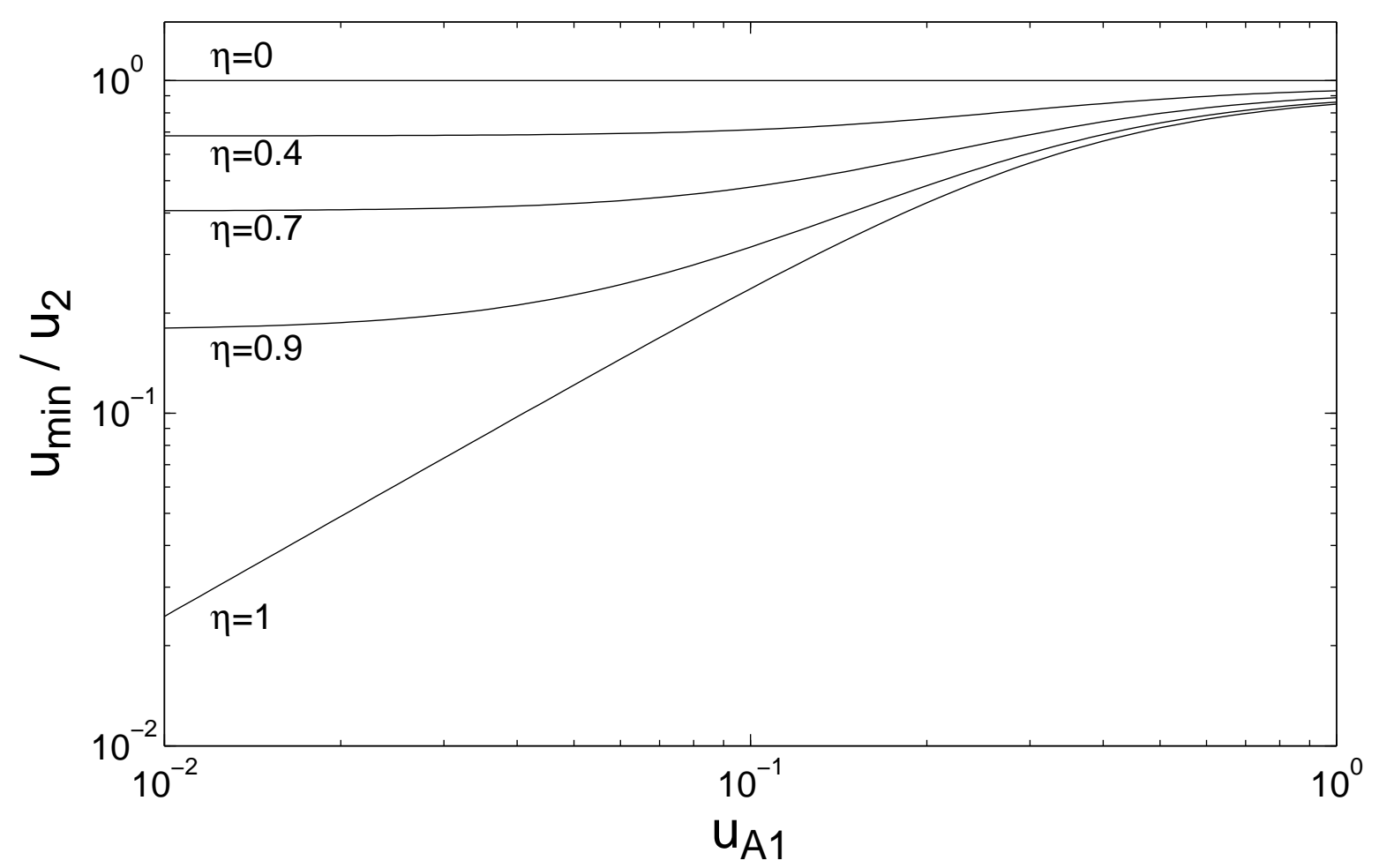

Fig. 3.- The asymptotic proper speed $u_{\min }$ (in units of $u_{2}$ ) as a function of $u_{A 1}$ for several values of $\eta$. 


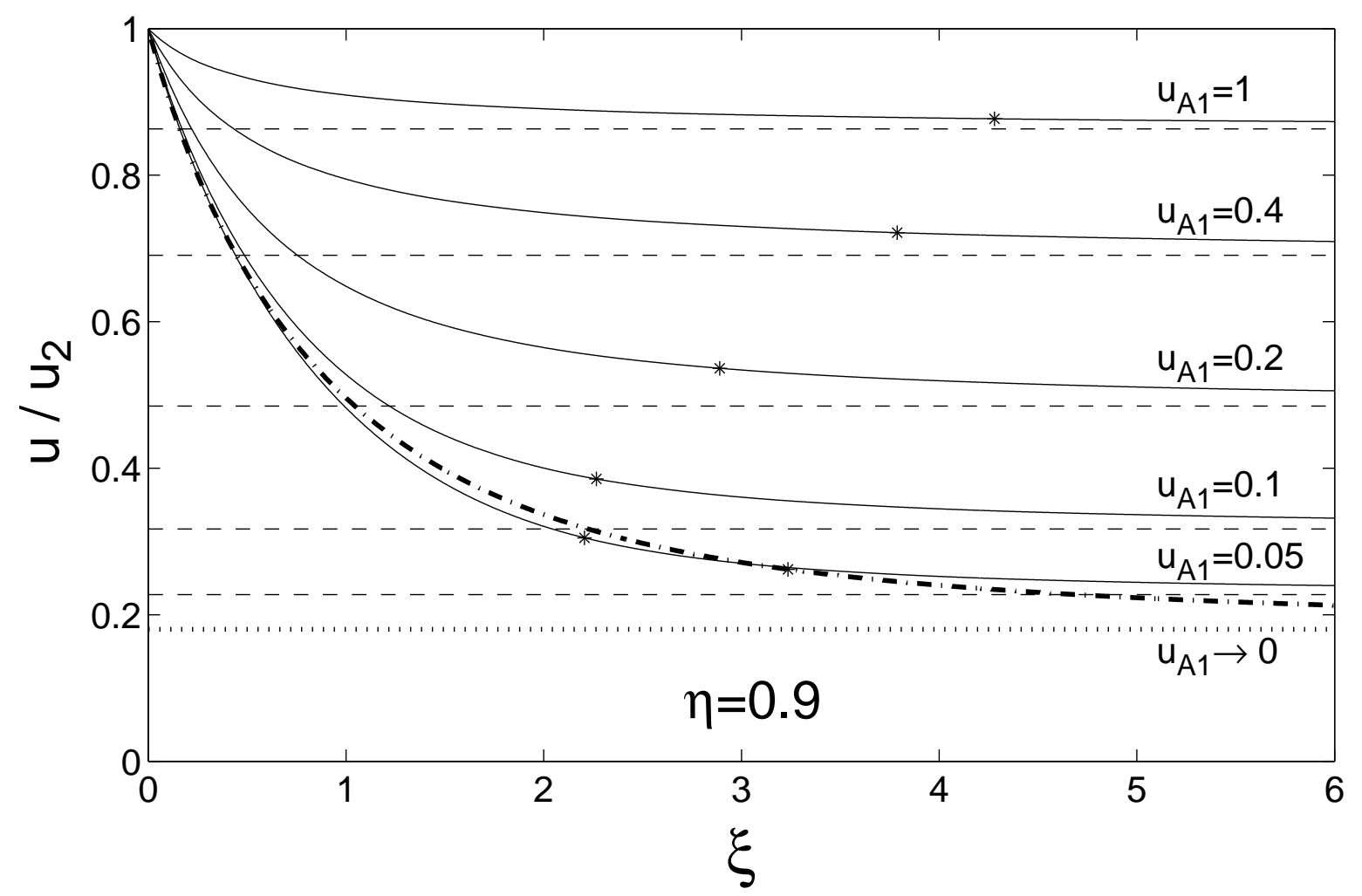

Fig. 4. - The proper speed $u$, normalized by the postshock value $u_{2}$, as a function of the normalized distance $\xi \equiv x / x_{c}$ for $\eta=0.9, b=0$, and several values of $u_{A 1}$ (solid lines). The dashed lines represent the corresponding asymptotic values $\left(u_{\min }\right)$. The boldface dash-dotted line depicts the limit $u_{A 1} \rightarrow 0$, and the boldface dotted line is the corresponding asymptotic value. An asterisk is placed on the solid lines and on the dash-dotted line at the point where $u$ has been reduced to $90 \%$ of its asymptotic value: $\left(u-u_{\min }\right) /\left(u_{2}-u_{\min }\right)=0.1$. 


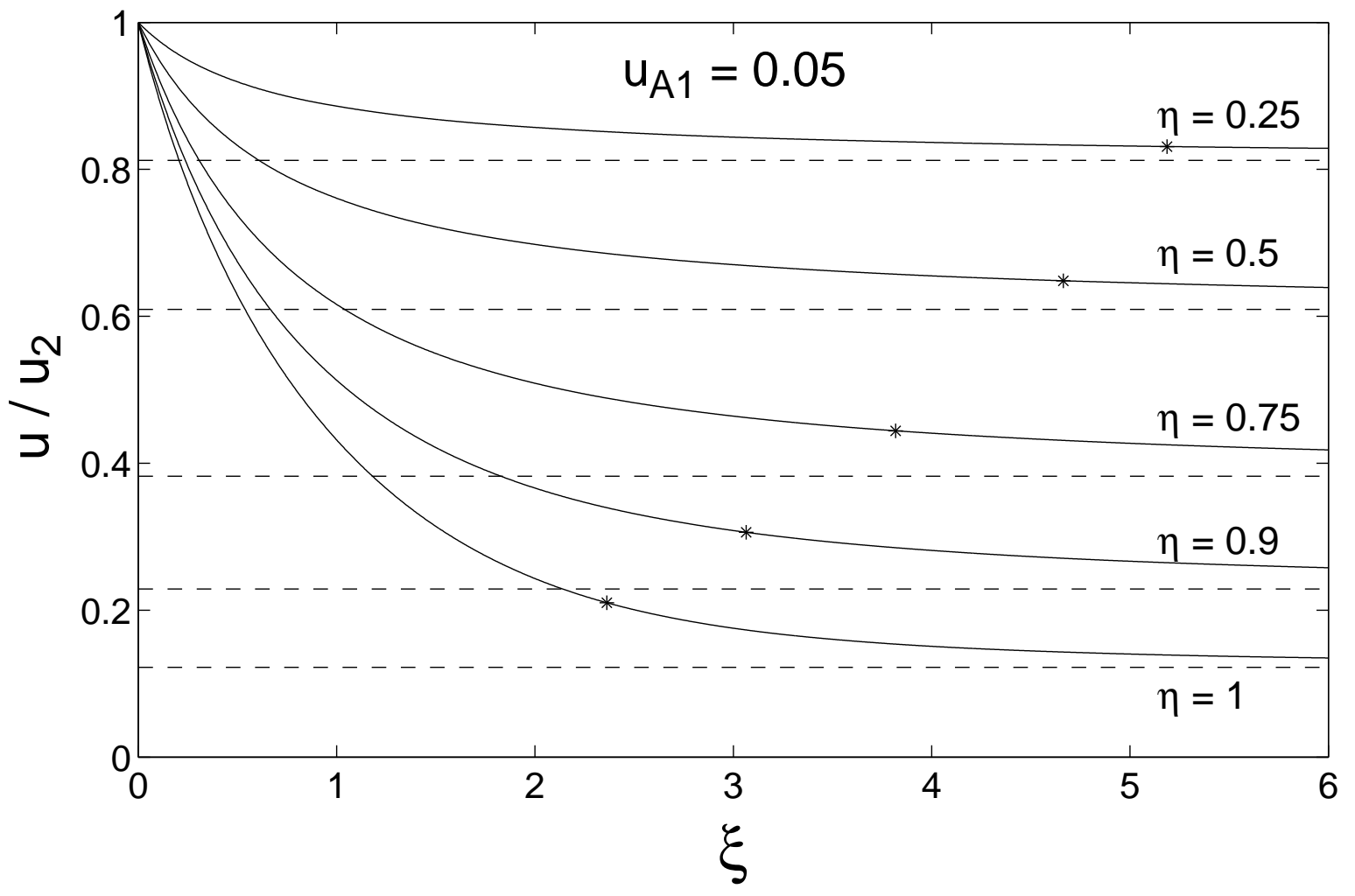

Fig. 5.- The proper speed $u$, normalized by the postshock value $u_{2}$, as a function of the normalized distance $\xi \equiv x / x_{c}$ for $u_{A 1}=0.05, b=0$, and several values of $\eta$ (solid lines). The dashed lines and the asterisk symbols have the same meaning as in Fig. $₫$. 


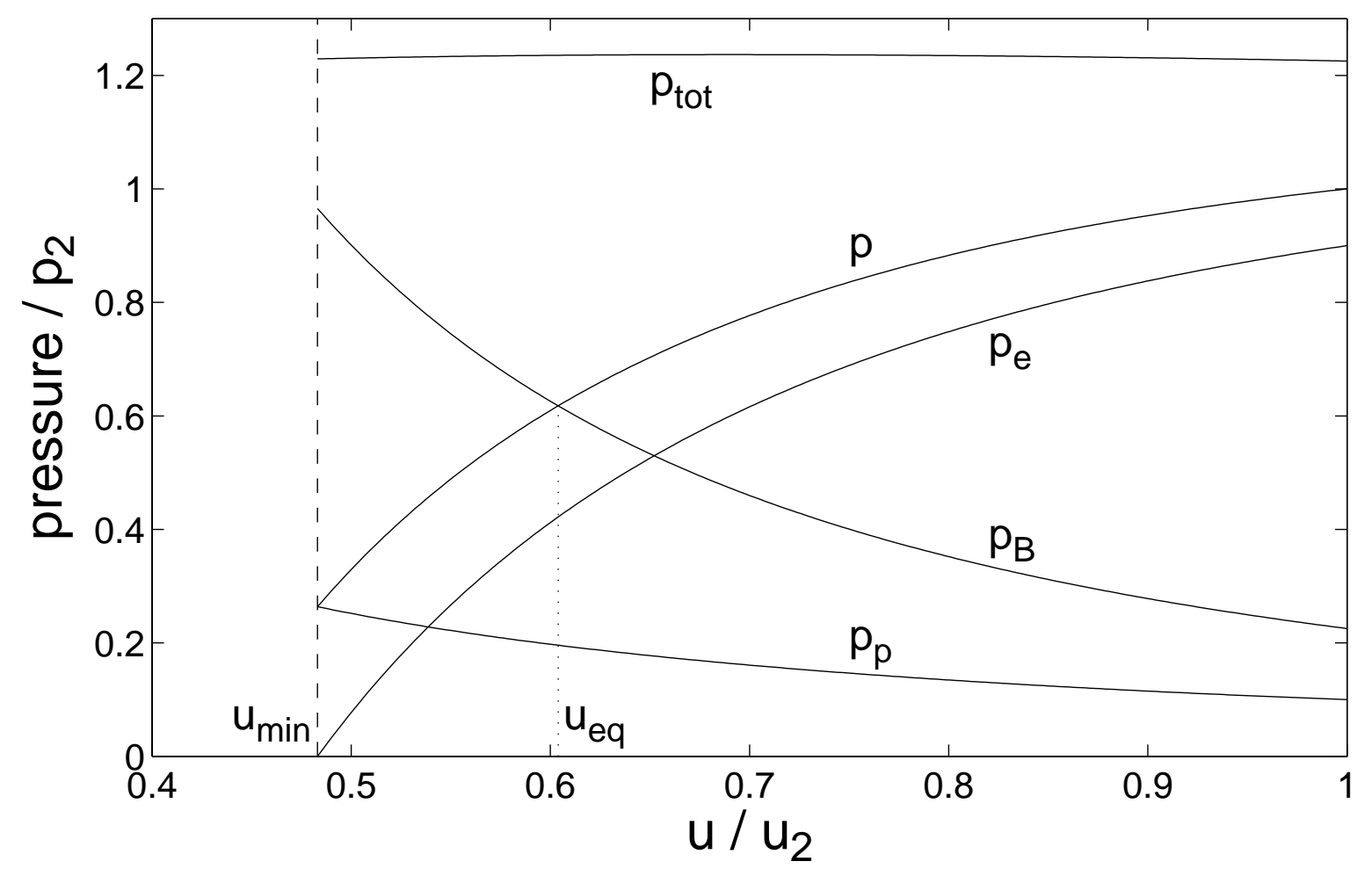

Fig. 6.- The pressures of the electron/positron $\left(p_{e}\right)$ and proton $\left(p_{p}\right)$ components, the total thermal pressure $\left(p=p_{e}+p_{p}\right)$, the magnetic field pressure $\left(p_{B}=B^{2} / 8 \pi\right)$ and the total pressure $\left(p_{\text {tot }}=p+p_{B}\right)$, all normalized by the postshock thermal pressure $p_{2}$, as a function of the normalized proper speed $u / u_{2}$ for $\eta=0.9$ and $u_{A 1}=0.2$. 


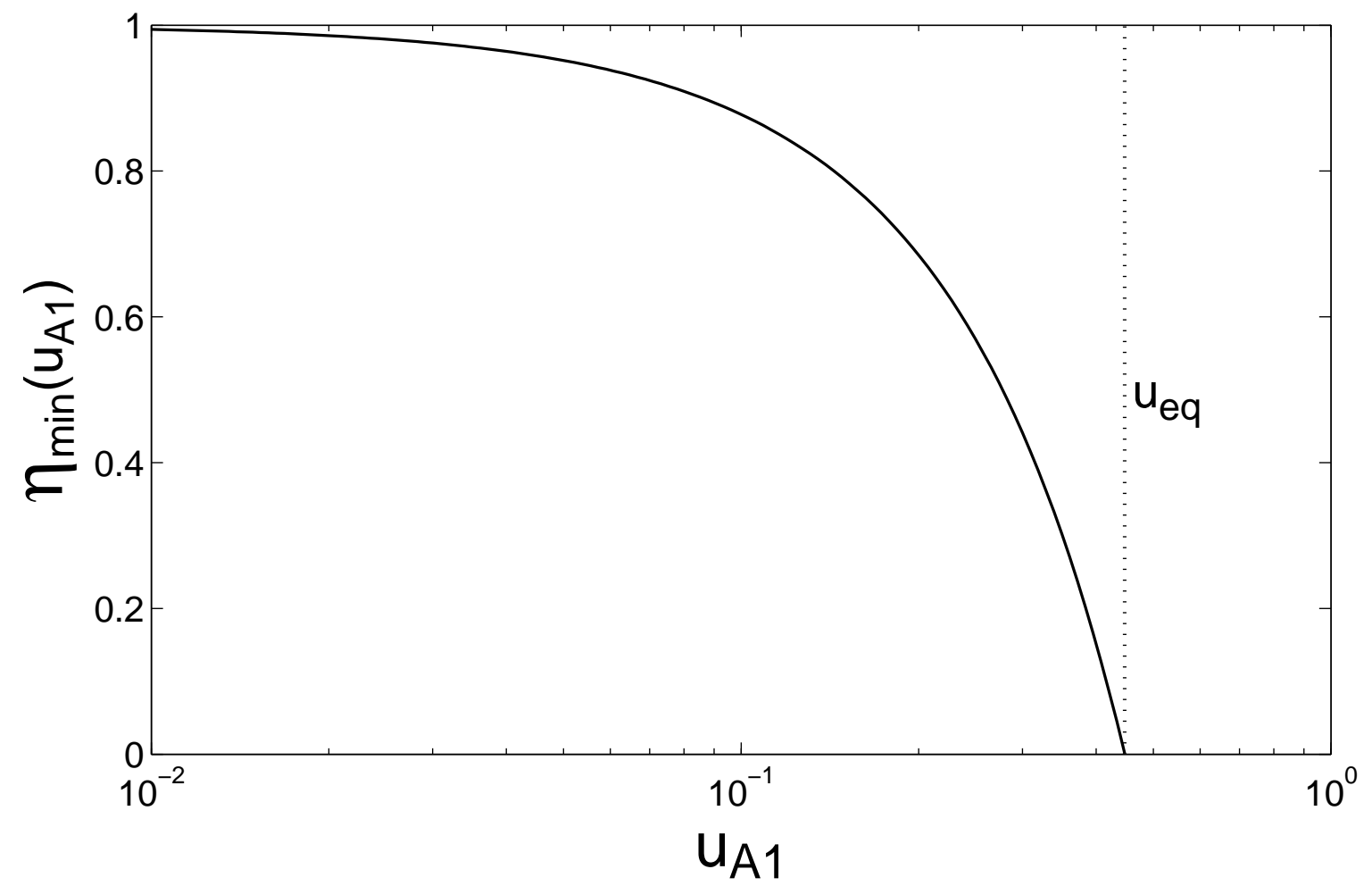

Fig. 7.- The minimum value of $\eta$ for which the magnetic field in the radiative zone reaches equipartition with the total thermal pressure, as a function of $u_{A 1}$. For $u_{A 1}>u_{\text {eq }}$ the magnetic field amplitude just behind the shock transition is already above equipartition. 


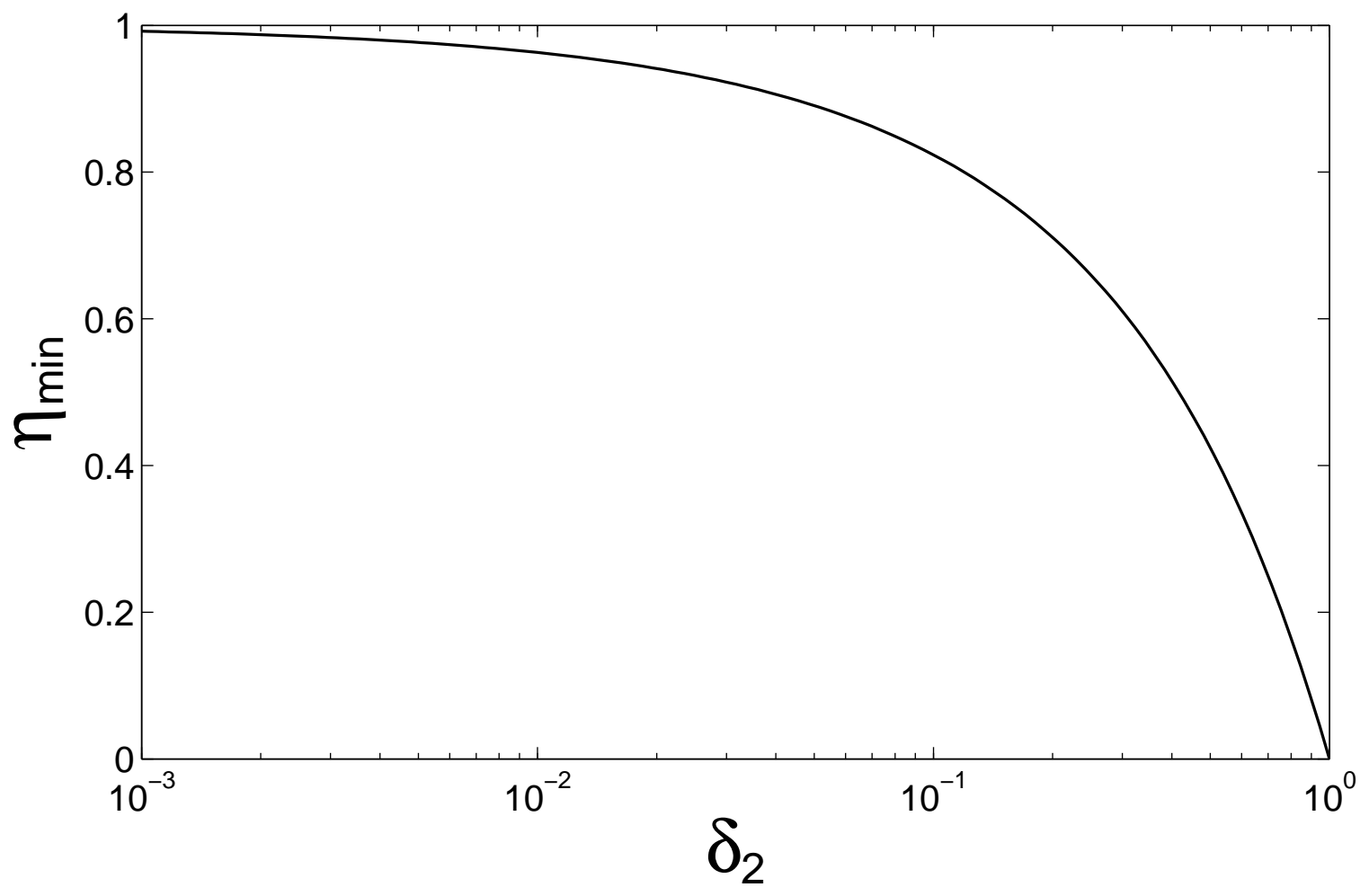

Fig. 8. - The minimum value of $\eta$ for which the magnetic field in the radiative zone reaches equipartition with the total thermal pressure, as a function of $\delta_{2}$, the ratio of the postshock magnetic pressure to its local equipartition value. 


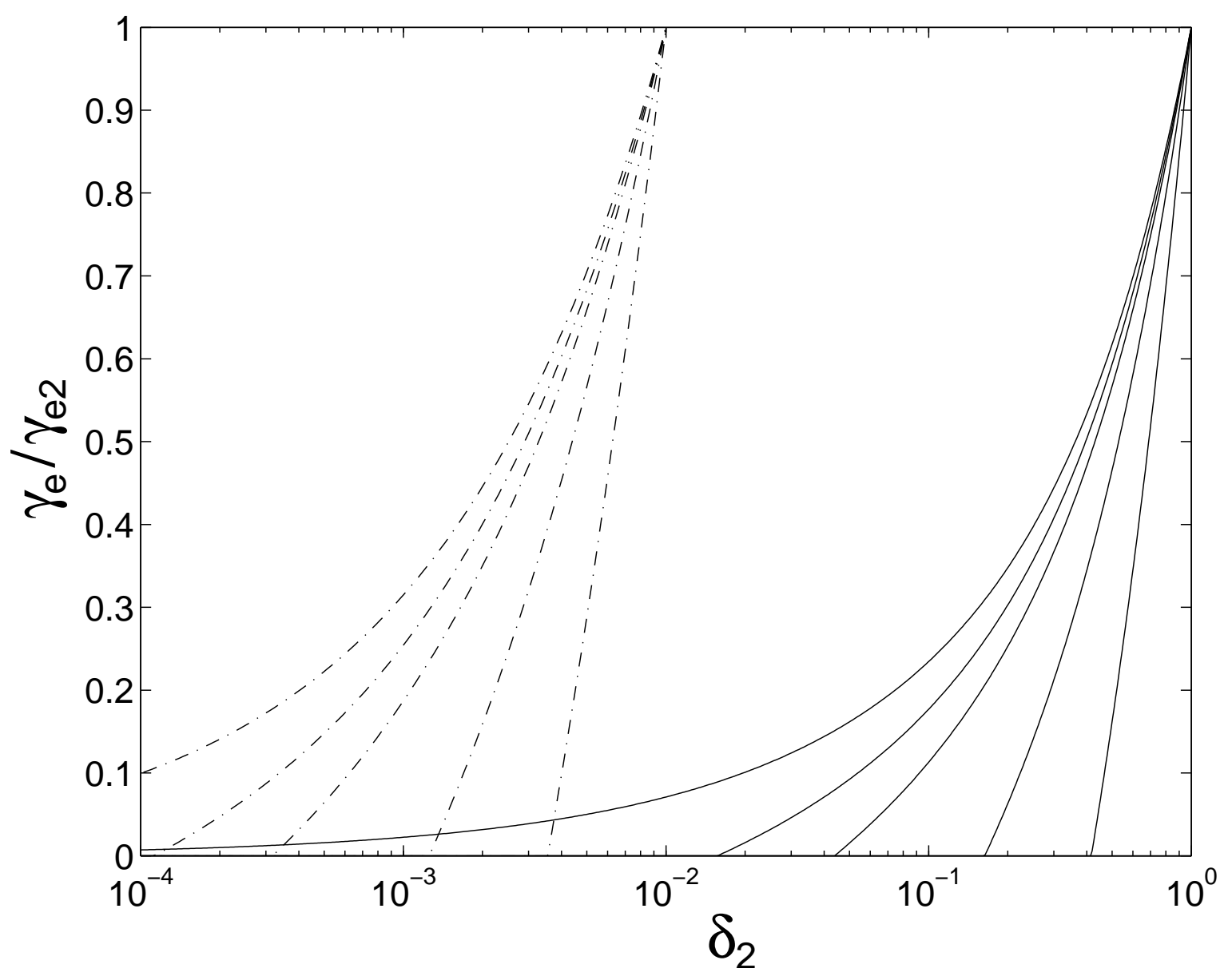

Fig. 9.- The fraction of the postshock internal energy still left in the electron/positron component as a function of the postshock magnetic-to-thermal pressure ratio, $\delta_{2}$ : (i) at the point where the magnetic field attains equipartition with the thermal pressure, $\delta=1$ (solid curves), and (ii) at the point where the magnetic pressure is $1 \%$ of the thermal pressure, $\delta=0.01$ (dash-dotted curves). The curves in each of the two cases correspond to $\eta=0.5,0.75,0.9,0.95$, and 1 , respectively, from right to left. 


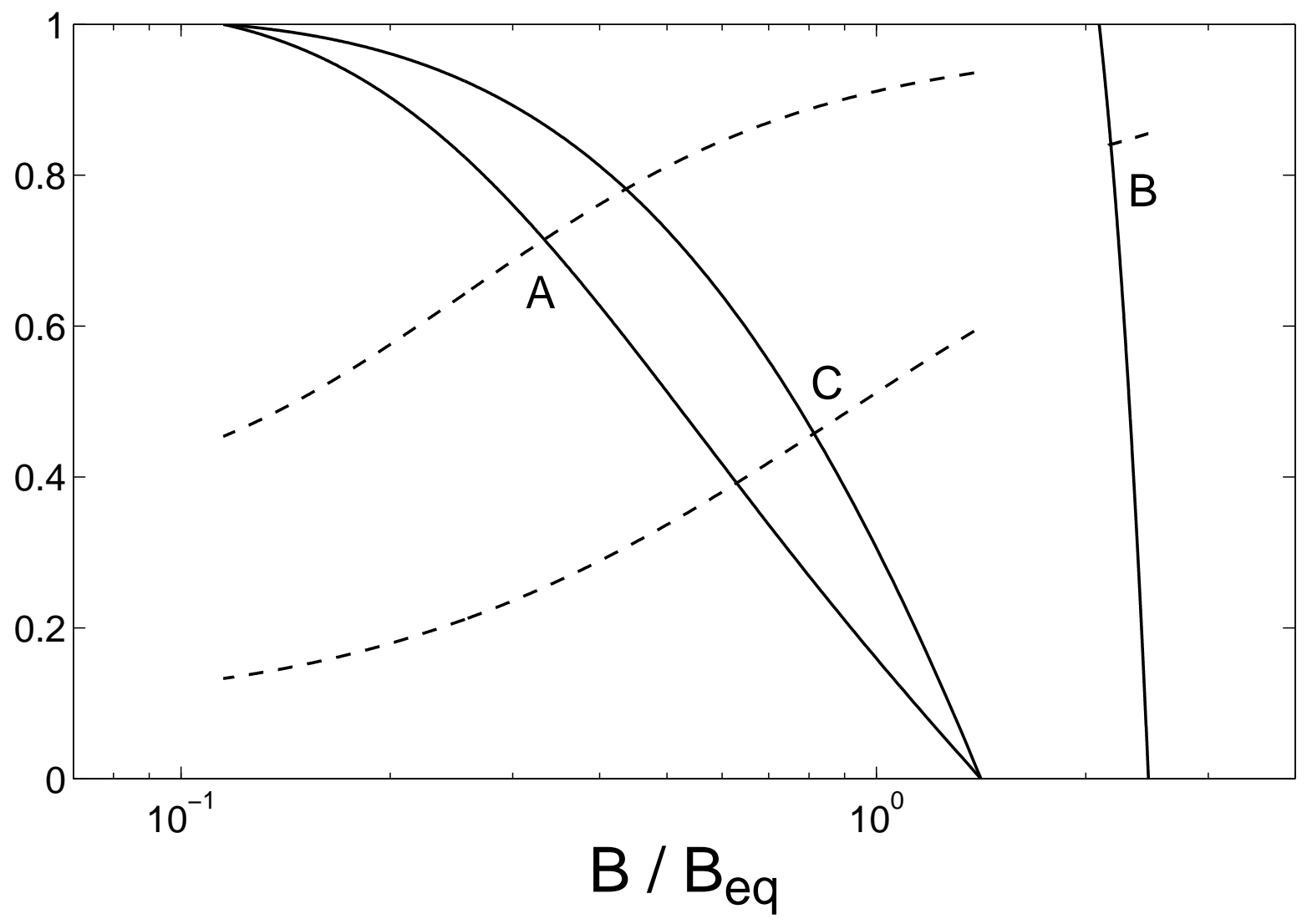

Fig. 10. - The solid lines show the fraction of the synchrotron emission that is emitted in a region where the magnetic field is larger than a given value, $P_{\text {syn }}(>B) / P_{\text {syn }}($ total), as a function of the magnetic field strength (normalized by its equipartition value). The dashed lines show the fraction of the total emission that is contributed by the synchrotron process, $P_{\text {syn }}(>B) / P_{\text {total }}(>B)$. The results are shown for three sets of parameters: $(\mathrm{A}) b=0, \eta=1$, $u_{A 1}=0.03$; (B) $b=0, \eta=1, u_{A 1}=1$; (C) $b=100, \eta=1, u_{A 1}=0.03$. 